ESAIM: M2AN 51 (2017) 2069-2092

DOI: $10.1051 / \mathrm{m} 2 \mathrm{an} / 2017015$
ESAIM: Mathematical Modelling and Numerical Analysis

www.esaim-m2an.org

\title{
HIGHER ORDER TOPOLOGICAL DERIVATIVES FOR THREE-DIMENSIONAL ANISOTROPIC ELASTICITY
}

\author{
Marc Bonnet ${ }^{1}$ And RÉmi Cornaggia ${ }^{2}$
}

\begin{abstract}
This article concerns an extension of the topological derivative concept for 3D elasticity problems involving elastic inhomogeneities, whereby an objective function $\mathbb{J}$ is expanded in powers of the characteristic size $a$ of a single small inhomogeneity. The $O\left(a^{6}\right)$ approximation of $\mathbb{J}$ is derived and justified for an inhomogeneity of given location, shape and elastic properties embedded in a 3D solid of arbitrary shape and elastic properties; the background and the inhomogeneity materials may both be anisotropic. The generalization to multiple small inhomogeneities is concisely described. Computational issues, and examples of objective functions commonly used in solid mechanics, are discussed.
\end{abstract}

Mathematics Subject Classification. 35C20, 45F15, 74B05.

Received May 26, 2016. Revised February 14, 2017. Accepted March 28, 2017.

\section{INTRODUCTION}

Many practical (e.g. design or identification) problems of physics or engineering involve the optimization of some objective function, whose definition often depends on the solution of a system of partial differential equations (PDE). The resulting need for efficient computational procedures for such PDE-constrained optimization problems has in turn prompted the appearance and implementation of many treatments, involving e.g. parameter or shape sensitivity, model reduction, or surrogate approximate models based on asymptotic methods. The latter category in particular includes the concept of topological derivative, which quantifies the perturbation induced to a cost functional $\mathbb{J}$ by the virtual creation of an inhomogeneity occupying a region $B_{a}(\boldsymbol{z})$ with a vanishingly small characteristic radius $a$ and a prescribed center located $\boldsymbol{z}$ inside the medium of interest. For the case of a small elastic inhomogeneity embedded in a three-dimensional elastic solid, considered in this work, the well known expansion

$$
\mathbb{J}\left(\mathcal{C}_{a}\right)=\mathbb{J}(\mathcal{C})+a^{3} \mathcal{T}(\boldsymbol{z})+o\left(a^{3}\right)
$$

holds $[13,21,35]$, where $\mathbb{J}\left(\mathcal{C}_{a}\right)$ denotes the value taken by the objective function of interest when the elastic properties of the perturbed solid are described by the piecewise-constant elasticity tensor field $\mathcal{C}_{a}$, such that $\operatorname{supp}\left(\mathcal{C}_{a}-\mathcal{C}\right)=B_{a}(\boldsymbol{z})(\mathcal{C}$ denoting the constant elasticity tensor for the background material). The topological derivative $\mathcal{T}(\boldsymbol{z})$, which depends on the location $\boldsymbol{z}$ of the trial inhomogeneity and also on its shape and on the elastic constants of both materials, can then be used e.g. to direct structural optimization algorithm

Keywords and phrases. Topological derivative, asymptotic expansion, volume integral equation, elastostatics.

1 POEMS (ENSTA ParisTech, CNRS, INRIA, Université Paris-Saclay), Palaiseau, France. mbonnet@ensta.fr

2 IRMAR, Université Rennes-1, Rennes, France. remi.cornaggia@univ-rennes1.fr 
towards optimal topologies, see e.g. $[1,6,33]$, or for qualitative flaw identification, see e.g. $[3,9,10,28]$. Objective functionals $\mathbb{J}$ used in such contexts depend on $\mathcal{C}_{a}$ implicitly through the displacement field $\boldsymbol{u}_{a}$ arising in the perturbed medium, i.e. have the form $\mathbb{J}\left(\mathcal{C}_{a}\right)=J\left(\boldsymbol{u}_{a}\right)$. The displacement perturbation $\boldsymbol{v}_{a}:=\boldsymbol{u}_{a}-\boldsymbol{u}(\boldsymbol{u}$ being the displacement in the unperturbed medium undergoing the same applied excitation) is well known from previous studies $[5,13]$ to verify $\boldsymbol{v}_{a}(\boldsymbol{x})=O\left(a^{3}\right)$ for any $\boldsymbol{x} \in \Omega, \boldsymbol{x} \neq \boldsymbol{z}$.

A natural extension of the concept of topological derivative consists in expanding $\mathbb{J}\left(\mathcal{C}_{a}\right)$ to higher orders in $a$, in order to formulate more accurate asymptotic approximations of $\mathbb{J}$. Previous efforts in this direction include [12] for inclusions or cracks in 2D conductive media, [11] for sound-hard obstacles in 3D acoustic media, and [34] for expansions of the potential energy of 2D elastic solids. Higher-order topological expansions were in particular shown in $[11,12]$ to allow computationally fast and quantitatively accurate flaw identification while avoiding the need for initial guesses.

This article, which is a sequel of [11-13], is devoted to establishing higher-order topological expansions of objective functionals for the case of a small elastic inhomogeneity embedded in a three-dimensional elastic solid. Such expansions have the form

$$
\mathbb{J}\left(\mathcal{C}_{a}\right)=\mathbb{J}(\mathcal{C})+a^{3} \mathcal{T}_{3}(\boldsymbol{z})+a^{4} \mathcal{T}_{4}(\boldsymbol{z})+a^{5} \mathcal{T}_{5}(\boldsymbol{z})+a^{6} \mathcal{T}_{6}(\boldsymbol{z})+o\left(a^{6}\right),
$$

involving higher-order topological derivatives $\mathcal{T}_{4}(\boldsymbol{z}), \mathcal{T}_{5}(\boldsymbol{z}), \mathcal{T}_{6}(\boldsymbol{z})$ in addition to the already-known ordinary topological derivative $\mathcal{T}_{3}(\boldsymbol{z})=\mathcal{T}(\boldsymbol{z})$. The distinguishing features of this work are as follows:

(a) The main result is an expansion having the form (1.1) for inhomogeneities of arbitrary shape, with both elastic materials permitted to be anisotropic, and for a large class of objective functionals. The chosen order $O\left(a^{6}\right)$ results from considering the minimization of least-squares cost functionals having the form $J\left(\boldsymbol{u}_{a}\right)=$ $\int\left|\boldsymbol{u}_{a}-\boldsymbol{u}^{\mathrm{m}}\right|^{2}=\int\left|\boldsymbol{u}+\boldsymbol{v}_{a}-\boldsymbol{u}^{\mathrm{m}}\right|^{2}$ with given $\boldsymbol{u}^{\mathrm{m}}$. In such cases, $a^{6} \mathcal{T}_{6}(\boldsymbol{z})$ is the lowest order term of expansion (1.1) to which the quadratic terms in $\boldsymbol{v}_{a}$ contribute. Analytical solutions for simple configurations, and evidence from previous numerical experiments on flaw identification problems, have shown that in practice we often have $\mathcal{T}_{6}(\boldsymbol{z})>0$ (thanks to the positive contribution of the second-order derivative $J^{\prime \prime}\left(\boldsymbol{u} ; \boldsymbol{v}_{a}\right)$ ) but $\mathcal{T}_{5}(\boldsymbol{z})<0$, implying that the $O\left(a^{6}\right)$ approximation $(1.1)$ of $\mathbb{J}\left(\mathcal{C}_{a}\right)$ has a minimum for $a \in \mathbb{R}^{+}$whereas the $O\left(a^{5}\right)$ approximation does not. We both derive the relevant high-order topological expansion (supplementing previously-known results on $\mathcal{T}_{3}(\boldsymbol{z})$ by giving complete expressions for functions $\mathcal{T}_{4}(\boldsymbol{z}), \mathcal{T}_{5}(\boldsymbol{z}), \mathcal{T}_{6}(\boldsymbol{z})$ ) and provide its justification; the previously-mentioned investigations $[11,12,34]$ addressed neither the general three-dimensional elastic case nor the justification of expansions derived and implemented therein.

(b) Like in $[11,12]$, we introduce the adjoint solution associated with $\mathbb{J}$ prior to performing the expansion in powers of $a$, allowing to establish the above expansion on the basis of (i) the expansion of the solution to the underlying elastic transmission problem in $B_{a}$ (higher-order inner expansion) and (ii) the leading-order contribution to the solution on the support of the objective function density (leading outer expansion).

(c) Setting up the expansion (1.1) requires information on the asymptotic behavior of the elastic transmission solution. The latter has been the subject of previous studies $[4,5]$ based on coupled boundary integral equation formulations. This work instead adopts a volume integral equation (VIE) framework, which is a natural setting for many inhomogeneity problems [19,27,36], including Eshelby's celebrated articles [17,18]. Here, the fact that the geometrical support of the VIE is $B_{a}$ facilitates the implementation and use of coordinate rescaling commonly used in the derivation of asymptotic models and allows, in combination with the adjoint solution approach, to focus the main efforts for both the derivation and the justification stage on the inner part of the solution expansion (i.e. that supported on $B_{a}$ ). Moreover, the VIE framework allows future extensions of this work to inhomogeneities with spatially-varying elastic properties.

We also mention that this work is a step towards setting up high-order topological expansions for the elastodynamic case, intended as a tool for the numerical solution of inverse scattering problem, in the spirit of [12].

This article is organised as follows. Section 2 describes the elastostatic problem and the class of cost functionals $\mathbb{J}$ undergoing expansion, introduces the governing volume integral equation (VIE) and the full-space 
transmission problems (FSTPs) that will serve as a basis for deriving the sought expansion, collects some facts about elastic moment tensors, and sets notation. Then, the governing VIE for the elastostatic transmission problem is expanded in Section 3, to obtain the first four terms of the inner expansion of the transmission solution and recovering the known leading-order outer solution expansion. Section 4 is devoted to the derivation and justification of the main result of this work, namely the $O\left(a^{6}\right)$ asymptotic expansion of $\mathbb{J}\left(\mathcal{C}_{a}\right)$ and the associated topological derivatives $\mathcal{T}_{3}(\boldsymbol{z}), \ldots, \mathcal{T}_{6}(\boldsymbol{z})$ for a single inhomogeneity of arbitrary shape and anisotropic elastic material embedded in a solid whose shape and (linearly elastic) material are also arbitrary (Theorem 4.1). Simpler formulas are then obtained for the common case of a centrally-symmetric inhomogeneity (Sect. 4.2), leading to explicit formulas for an ellipsoidal inhomogeneity (Sect. 4.3). Then, Section 5 is devoted to discussing some computational issues, specializing the results of Section 4 to commonly-used objective functionals, and briefly describing how Theorem 4.1 can be generalized to the case of multiple inhomogeneities with fixed locations. Finally, Sections 6 and 7 give the proof of two sub-results upon which Theorem 4.1 relies, namely the well-posedness of the VIE formulation of elastostatic FSTPs (Prop. 2.2) and the justification of the inner expansion of the transmission solution (Prop. 3.1).

\section{Elastostatic PRoblem And Cost FunCtional}

Consider a homogeneous reference solid body occupying the bounded Lipschitz domain $\Omega \subset \mathbb{R}^{3}$, with linearly elastic material behavior characterized by the (possibly anisotropic) elasticity tensor $\mathcal{C}$, which is symmetric and positive definite as a linear operator acting on $\mathbb{R}_{\mathrm{sym}}^{3 \times 3}$; moreover, $\mathcal{C}$ is also assumed to be bounded (thereby excluding the case of incompressible background materials). When the material is isotropic, $\mathcal{C}$ is given by

$$
\mathcal{C}=2 \mu\left(\frac{1+\nu}{1-2 \nu} \mathcal{J}+\mathcal{K}\right)
$$

where $\mu>0$ is the shear modulus and $0<\nu<\frac{1}{2}$ is Poisson's ratio ${ }^{3}$. The fourth-order tensors $\mathcal{J}, \mathcal{K}$ are respectively defined by $\mathcal{J}=(1 / 3) \boldsymbol{I} \otimes \boldsymbol{I}$ and $\mathcal{K}=\mathcal{I}-\mathcal{J}$ in terms of the second-order identity $\boldsymbol{I}$ (with $I_{i j}=\delta_{i j}$ ) and the fourth-order identity for symmetric tensors $\mathcal{I}\left(\right.$ with $\mathcal{I}_{i j k \ell}=\frac{1}{2}\left(\delta_{i k} \delta_{j \ell}+\delta_{i \ell} \delta_{j k}\right)$ ).

The solid is subjected to prescribed displacements $\boldsymbol{u}^{\mathrm{D}}$ and surface force densities $\boldsymbol{t}^{\mathrm{D}}$, respectively applied on the surfaces $\Gamma_{\mathrm{D}}$ and $\Gamma_{\mathrm{N}}$ such that $\Gamma_{\mathrm{D}} \cup \Gamma_{\mathrm{N}}=\partial \Omega, \Gamma_{\mathrm{D}} \cap \Gamma_{\mathrm{N}}=\emptyset$ and $\left|\Gamma_{\mathrm{D}}\right| \neq 0$. These excitations (which are chosen for definiteness, as other choices are possible, see Rem. 2.1) give rise to the background displacement field $\boldsymbol{u} \in \boldsymbol{H}^{1}(\Omega)$ satisfying:

$$
\operatorname{div}(\mathcal{C}: \varepsilon[\boldsymbol{u}])=\mathbf{0} \text { in } \Omega, \quad \boldsymbol{u}=\boldsymbol{u}^{\mathrm{D}} \text { on } \Gamma_{\mathrm{D}}, \quad \boldsymbol{t}[\boldsymbol{u}]=\boldsymbol{t}^{\mathrm{D}} \text { on } \Gamma_{\mathrm{N}},
$$

where $\varepsilon[\boldsymbol{w}]$ and $\boldsymbol{t}[\boldsymbol{w}]$ denote the linearized strain tensor and the traction vector associated with a given displacement $\boldsymbol{w}$, respectively defined by

$$
\text { (a) } \varepsilon[\boldsymbol{w}]=\frac{1}{2}\left(\boldsymbol{\nabla} \boldsymbol{w}+(\boldsymbol{\nabla} \boldsymbol{w})^{\mathrm{T}}\right), \quad \text { (b) } \boldsymbol{t}[\boldsymbol{w}]=(\mathcal{C}: \varepsilon[\boldsymbol{w}]) \cdot \boldsymbol{n}
$$

(with $\boldsymbol{n}$ the unit outward normal to $\Omega$ ). In (2.2) and hereinafter, symbols '.' and ':' denote single and double inner products, e.g. $(\boldsymbol{E} \cdot \boldsymbol{x})_{i}=E_{i j} x_{j}$ and $(\mathcal{C}: \boldsymbol{E})_{i j}=\mathcal{C}_{i j k \ell} E_{k \ell}$, with Einstein's convention of summation over repeated indices implicitly used throughout; moreover, boldface symbols $\boldsymbol{H}^{1}, \boldsymbol{L}^{2}$ indicate classical Sobolev spaces of vector- or tensor-valued fields (depending on context), e.g. $\boldsymbol{H}^{1}(X)=H^{1}\left(X ; \mathbb{R}^{3}\right)$ for some domain $X \subset \mathbb{R}^{3}$.

The weak formulation corresponding to problem (2.2) is:

$$
\text { Find } \boldsymbol{u} \in \mathcal{V}, \quad\langle\boldsymbol{u}, \boldsymbol{w}\rangle_{\Omega}^{\mathcal{C}}=F(\boldsymbol{w}), \quad \forall \boldsymbol{w} \in \mathcal{V}_{0},
$$

\footnotetext{
${ }^{3}$ The range $0<\nu<\frac{1}{2}$ corresponds to "usual" isotropic materials. The elasticity system is strongly elliptic for $\nu \in \mathbb{R} \backslash[1 / 2,1]$, while the strain energy is positive definite for $-1<\nu<\frac{1}{2}$. Certain microstructured materials achieve $-1<\nu<0$. See e.g. [37].
} 
where the notation $\langle\boldsymbol{u}, \boldsymbol{w}\rangle_{D}^{\mathcal{C}}$, used throughout this article, stands for the bilinear elastic energy form associated to given domain $D \subset \mathbb{R}^{3}$, elasticity tensor $\mathcal{C}$ and displacement fields $\boldsymbol{u}, \boldsymbol{w}$, i.e.:

$$
\langle\boldsymbol{u}, \boldsymbol{w}\rangle_{D}^{\mathcal{C}}:=\int_{D} \varepsilon[\boldsymbol{u}]: \mathcal{C}: \varepsilon[\boldsymbol{w}] \mathrm{d} V=\int_{D} \boldsymbol{\nabla} \boldsymbol{u}: \mathcal{C}: \boldsymbol{\nabla} \boldsymbol{w} \mathrm{d} V
$$

(the second equality resulting from the minor symmetries of $\mathcal{C}$ ), the linear form $F$ associated to the loading is defined by

$$
F(\boldsymbol{w})=\int_{\Gamma_{\mathrm{N}}} \boldsymbol{t}^{\mathrm{D}} \cdot \boldsymbol{w} \mathrm{d} S
$$

and the spaces $\mathcal{V}, \mathcal{V}_{0}$ are $\mathcal{V}=\left\{\boldsymbol{u} \in \boldsymbol{H}^{1}(\Omega), \boldsymbol{u}=\boldsymbol{u}^{\mathrm{D}}\right.$ on $\left.\Gamma_{\mathrm{D}}\right\}, \mathcal{V}_{0}=\left\{\boldsymbol{u} \in \boldsymbol{H}^{1}(\Omega), \boldsymbol{u}=\mathbf{0}\right.$ on $\left.\Gamma_{\mathrm{D}}\right\}$.

\subsection{Transmission by a small inhomogeneity}

An inhomogeneity $B_{a}$ of small size $a$ and shape $\mathcal{B}$ is then introduced in the medium, centered at a point $\boldsymbol{z}$, so that we note $B_{a}=\boldsymbol{z}+a \mathcal{B}$. It is characterized by the homogeneous elasticity tensor $\mathcal{C}^{\star}$, also assumed to be positive definite and bounded (thereby excluding voids, for which $\mathcal{C}^{\star}=\mathbf{0}$, and incompressible inhomogeneities). If isotropic, $\mathcal{C}^{\star}$ is of the form $(2.1)$ with parameters $\mu^{\star}>0,0<\nu^{\star}<\frac{1}{2}$. We set $\Delta \mathcal{C}:=\mathcal{C}^{\star}-\mathcal{C}$. By analogy with (2.4), the displacement $\boldsymbol{u}_{a}$ for the perturbed solid solves the weak formulation

$$
\text { Find } \boldsymbol{u}_{a} \in \mathcal{V}, \quad\left\langle\boldsymbol{u}_{a}, \boldsymbol{w}\right\rangle_{\Omega}^{\mathcal{C}_{a}}=F(\boldsymbol{w}), \quad \forall \boldsymbol{w} \in \mathcal{V}_{0},
$$

where $\mathcal{C}_{a}=\mathcal{C}+\chi\left(B_{a}\right) \Delta \mathcal{C}$ is the (piecewise-constant) elasticity tensor field for the whole solid $(\chi(D)$ being the characteristic function of a domain $D \subset \mathbb{R}^{3}$ ). Substracting (2.4) from (2.6), the perturbation displacement $\boldsymbol{v}_{a}:=\boldsymbol{u}_{a}-\boldsymbol{u}$ is found to solve the weak formulation:

$$
\text { Find } \boldsymbol{v}_{a} \in \mathcal{V}_{0}, \quad\left\langle\boldsymbol{v}_{a}, \boldsymbol{w}\right\rangle_{\Omega}^{\mathcal{C}_{a}}=-\langle\boldsymbol{u}, \boldsymbol{w}\rangle_{B_{a}}^{\Delta \mathcal{C}}, \quad \forall \boldsymbol{w} \in \mathcal{V}_{0}
$$

Remark 2.1. The excitation used in the definition (2.2) of the background displacement is chosen for the sake of definiteness. Other types of excitations involving e.g. body forces, initial strains or stresses are also permissible, provided they allow sufficient $\left(C^{5}\right.$, see proof of Thm. 4.1) interior regularity of $\boldsymbol{u}$ at the chosen inhomogeneity site $\boldsymbol{z}$ (i.e. either $\boldsymbol{z}$ is outside the support of any internal excitation, or the latter has enough smoothness at $\boldsymbol{z}$ ).

\subsection{Cost functional and adjoint problem}

We consider objective functionals $\mathbb{J}\left(\mathcal{C}_{a}\right)$ that depend on the trial inhomogeneity (i.e. on $\left.\mathcal{C}_{a}\right)$ implicitly through the solution $\boldsymbol{u}_{a}$ of (2.6), of the form

$$
\mathbb{J}\left(\mathcal{C}_{a}\right)=J\left(\boldsymbol{u}_{a}\right), \quad \text { with } \quad J(\boldsymbol{w})=\int_{\Omega^{\mathrm{m}}} \Psi_{\Omega}(\boldsymbol{x}, \boldsymbol{w}(\boldsymbol{x})) \mathrm{d} V_{x}+\int_{\Gamma^{\mathrm{m}}} \Psi_{\Gamma}(\boldsymbol{x}, \boldsymbol{w}(\boldsymbol{x})) \mathrm{d} S_{x},
$$

with the volume and surface densities $\Psi_{\Omega}:\left(\Omega^{\mathrm{m}} \times \mathbb{R}^{3}\right) \rightarrow \mathbb{R}$ and $\Psi_{\Gamma}:\left(\Gamma^{\mathrm{m}} \times \mathbb{R}^{3}\right) \rightarrow \mathbb{R}$ assumed to be twice differentiable in their second argument; moreover, the corresponding second-order derivatives of $\Psi_{\Omega}$ and $\Psi_{\Gamma}$ are assumed to have $C^{0, \gamma}$ regularity with respect to their second argument for some $\gamma>0$. The supports $\Omega^{\mathrm{m}}, \Gamma^{\mathrm{m}}$ are open subsets of $\Omega$ and $\Gamma_{\mathrm{N}}$, respectively; moreover we assume that $\boldsymbol{z} \notin \Omega^{\mathrm{m}}$, implying that $B_{a} \cap \Omega^{\mathrm{m}}=\emptyset$ for $a$ small enough. Using Taylor expansions (with integral remainder) of $\Psi_{\Omega}, \Psi_{\Gamma}$, the following expansion of $J\left(\boldsymbol{u}_{a}\right)$ about the background solution $\boldsymbol{u}$ therefore holds:

$$
\mathbb{J}\left(\mathcal{C}_{a}\right)-\mathbb{J}(\mathcal{C})=J^{\prime}\left(\boldsymbol{u} ; \boldsymbol{v}_{a}\right)+\frac{1}{2} J^{\prime \prime}\left(\boldsymbol{u} ; \boldsymbol{v}_{a}\right)+R\left(\boldsymbol{u} ; \boldsymbol{v}_{a}\right)
$$


with

$$
\begin{aligned}
& J^{\prime}\left(\boldsymbol{u} ; \boldsymbol{v}_{a}\right)=\int_{\Omega^{\mathrm{m}}} \boldsymbol{\nabla}_{2} \Psi_{\Omega}(\cdot ; \boldsymbol{u}) \cdot \boldsymbol{v}_{a} \mathrm{~d} V+\int_{\Gamma^{\mathrm{m}}} \boldsymbol{\nabla}_{2} \Psi_{\Gamma}(\cdot ; \boldsymbol{u}) \cdot \boldsymbol{v}_{a} \mathrm{~d} S \\
& J^{\prime \prime}\left(\boldsymbol{u} ; \boldsymbol{v}_{a}\right)=\int_{\Omega^{\mathrm{m}}} \boldsymbol{\nabla}_{22} \Psi_{\Omega}(\cdot ; \boldsymbol{u}):\left(\boldsymbol{v}_{a} \otimes \boldsymbol{v}_{a}\right) \mathrm{d} V+\int_{\Gamma^{\mathrm{m}}} \boldsymbol{\nabla}_{22} \Psi_{\Gamma}(\cdot ; \boldsymbol{u}):\left(\boldsymbol{v}_{a} \otimes \boldsymbol{v}_{a}\right) \mathrm{d} S \\
& R\left(\boldsymbol{u} ; \boldsymbol{v}_{a}\right)=\int_{0}^{1}(1-t) J^{\prime \prime}\left(\boldsymbol{u}+t \boldsymbol{v}_{a} ; \boldsymbol{v}_{a}\right) \mathrm{d} t-\frac{1}{2} J^{\prime \prime}\left(\boldsymbol{u} ; \boldsymbol{v}_{a}\right)
\end{aligned}
$$

where, for any function $\psi$ having two arguments, $\nabla_{k} \psi$ denote its gradient with respect to its (possibly vectorvalued) $k$ th argument and $\nabla_{k \ell}$ its second-order gradient w.r.t. $k$ th and $\ell$ th arguments; moreover, the symbol $\otimes$ indicates the usual tensor product.

To evaluate the directional derivative $J^{\prime}\left(\boldsymbol{u} ; \boldsymbol{v}_{a}\right)$, it is convenient and customary to introduce the adjoint solution $\hat{\boldsymbol{u}}$ defined by the weak formulation

$$
\text { Find } \hat{\boldsymbol{u}} \in \mathcal{V}_{0}, \quad\langle\hat{\boldsymbol{u}}, \boldsymbol{w}\rangle_{\Omega}^{\mathcal{C}}=J^{\prime}(\boldsymbol{u} ; \boldsymbol{w}), \quad \forall \boldsymbol{w} \in \mathcal{V}_{0}
$$

Then, on setting $\boldsymbol{w}=\hat{\boldsymbol{u}}$ in (2.7) and $\boldsymbol{w}=\boldsymbol{v}_{a}$ in (2.10), combining the resulting identities and exploiting the symmetry of the energy bilinear form, one obtains the following reformulation of $J^{\prime}\left(\boldsymbol{u} ; \boldsymbol{v}_{a}\right)$ :

$$
J^{\prime}\left(\boldsymbol{u} ; \boldsymbol{v}_{a}\right)=-\langle\hat{\boldsymbol{u}}, \boldsymbol{u}\rangle_{B_{a}}^{\Delta \mathcal{C}}-\left\langle\hat{\boldsymbol{u}}, \boldsymbol{v}_{a}\right\rangle_{B_{a}}^{\Delta \mathcal{C}}=-\left\langle\hat{\boldsymbol{u}}, \boldsymbol{u}_{a}\right\rangle_{B_{a}}^{\Delta \mathcal{C}},
$$

which will facilitate subsequent asymptotic expansions by virtue of having $B_{a}$ as its support.

\subsubsection{Least-squares misfit cost functional.}

This simple but important type of cost functional is commonly used for e.g. defect identification. It evaluates the misfit between a given displacement $\boldsymbol{w}$ and experimental values $\boldsymbol{u}^{\mathrm{m}}$ recorded on a measurement surface $\Gamma^{\mathrm{m}} \subset \Gamma_{\mathrm{N}}$ :

$$
J(\boldsymbol{w})=\frac{1}{2} \int_{\Gamma^{\mathrm{m}}}\left|\boldsymbol{w}-\boldsymbol{u}^{\mathrm{m}}\right|^{2} \mathrm{~d} S
$$

corresponding to $\Psi_{\Gamma}(\boldsymbol{x}, \boldsymbol{w})=\frac{1}{2}\left|\boldsymbol{w}-\boldsymbol{u}^{\mathrm{m}}(\boldsymbol{x})\right|^{2}\left(\boldsymbol{x} \in \Gamma^{\mathrm{m}}\right)$. In this case, the expansion (2.9) is exact and holds with

$$
J^{\prime}\left(\boldsymbol{u} ; \boldsymbol{v}_{a}\right)=\int_{\Gamma^{\mathrm{m}}}\left(\boldsymbol{u}-\boldsymbol{u}^{\mathrm{m}}\right) \cdot \boldsymbol{v}_{a} \mathrm{~d} S, \quad J^{\prime \prime}\left(\boldsymbol{u} ; \boldsymbol{v}_{a}\right)=\int_{\Gamma_{\mathrm{m}}}\left|\boldsymbol{v}_{a}\right|^{2} \mathrm{~d} S, \quad R\left(\boldsymbol{u} ; \boldsymbol{v}_{a}\right)=0
$$

The case of interior measurements of displacements [23] recorded in a measurement region $\Omega^{\mathrm{m}} \subset \Omega$ can be accommodated in essentially identical fashion, setting $\Psi_{\Omega}(\boldsymbol{x}, \boldsymbol{w})=\frac{1}{2}\left|\boldsymbol{w}-\boldsymbol{u}^{\mathrm{m}}(\boldsymbol{x})\right|^{2}\left(\boldsymbol{x} \in \Omega^{\mathrm{m}}\right)$.

\subsection{Small-inhomogeneity expansion}

As mentioned in the Introduction, it is well known from previous studies that $\boldsymbol{v}_{a}(\boldsymbol{x})=O\left(a^{3}\right)$ as $a \rightarrow 0$ for any fixed point $\boldsymbol{x} \neq \boldsymbol{z}$. Therefore, if the expansion of $\mathbb{J}\left(\mathcal{C}_{a}\right)$ is to include (as desired) the leading contribution as $a \rightarrow 0$ of $J^{\prime \prime}\left(u ; \boldsymbol{v}_{a}\right)$, it must be performed up to order $O\left(a^{6}\right)$ at least. Establishing the sought expansion of $\mathbb{J}\left(\mathcal{C}_{a}\right)$ in $a$ in turn necessitates an expansion of the transmission solution $\boldsymbol{v}_{a}$. Since $J^{\prime}\left(u, \boldsymbol{v}_{a}\right)$ is formulated in terms of integrals over the vanishing support $B_{a}$ (see (2.11)), finding the expansion of $\boldsymbol{v}_{a}$ will be facilitated by stating the transmission problem (2.7) as a volume integral equation (VIE) with support $B_{a}$, as shown now. 


\subsection{Transmission problem: volume integral equation formulation}

\subsubsection{Elastostatic Green's tensor}

Let $\boldsymbol{G}=\boldsymbol{e}_{k} \otimes \boldsymbol{G}^{k}$ be the elastostatic Green's tensor associated to problem (2.2), with each vector $\boldsymbol{G}^{k}(\boldsymbol{\xi}, \boldsymbol{x})$ solving, for a given source point $\boldsymbol{x} \in \Omega$, the problem

$$
\operatorname{div}\left(\mathcal{C}: \nabla_{1} \boldsymbol{G}^{k}(\cdot, \boldsymbol{x})\right)+\delta_{\boldsymbol{x}} \boldsymbol{e}_{k}=\mathbf{0} \quad \text { in } \Omega, \quad \boldsymbol{G}^{k}(\cdot, \boldsymbol{x})=\mathbf{0} \quad \text { on } \Gamma_{\mathrm{D}}, \quad \boldsymbol{t}\left[\boldsymbol{G}^{k}(\cdot, \boldsymbol{x})\right]=\mathbf{0} \quad \text { on } \Gamma_{\mathrm{N}}
$$

(where $\delta_{\boldsymbol{x}}$ denotes the Dirac distribution supported at $\boldsymbol{x}$, and the notation $\boldsymbol{\nabla}_{1} \boldsymbol{G}$ follows the convention introduced in (2.9) for functions having two arguments). The component $G_{i j}(\boldsymbol{\xi}, \boldsymbol{x})$ of $\boldsymbol{G}(\boldsymbol{\xi}, \boldsymbol{x})$ is therefore the $j$ th component of the displacement at $\boldsymbol{\xi}$ resulting from a unit point force applied at $\boldsymbol{x}$ along the $i$ th direction. The Green's tensor can then be decomposed according to:

$$
\boldsymbol{G}(\boldsymbol{\xi}, \boldsymbol{x})=\boldsymbol{G}_{\infty}(\boldsymbol{\xi}-\boldsymbol{x})+\boldsymbol{G}_{\mathrm{c}}(\boldsymbol{\xi}, \boldsymbol{x})
$$

where $\boldsymbol{G}_{\infty}=\boldsymbol{e}_{k} \otimes \boldsymbol{G}_{\infty}^{k}$ is the (singular) full-space Green's tensor (or fundamental tensor) satisfying

$$
\operatorname{div}\left(\mathcal{C}: \nabla G_{\infty}^{k}\right)+\delta \boldsymbol{e}_{k}=0 \quad \text { in } \quad \mathbb{R}^{3}, \quad\left|\boldsymbol{G}_{\infty}^{k}(\boldsymbol{r})\right| \rightarrow 0 \quad \text { as } \quad|\boldsymbol{r}| \rightarrow \infty
$$

while the complementary part $\boldsymbol{G}_{\mathrm{c}}(\cdot, \boldsymbol{x})=\boldsymbol{e}_{k} \otimes \boldsymbol{G}_{\mathrm{c}}^{k}(\cdot, \boldsymbol{x})$ is seen (using superposition) to be such that each $\boldsymbol{G}_{\mathrm{c}}^{k}$ solves an elastostatic boundary-value problem of the form (2.2) with boundary data $\boldsymbol{u}^{\mathrm{D}}=-\boldsymbol{G}_{\infty}^{k}(\cdot-\boldsymbol{x})$, $\boldsymbol{t}^{\mathrm{D}}=-\boldsymbol{t}\left[\boldsymbol{G}_{\infty}^{k}(\cdot-\boldsymbol{x})\right]$. Therefore, $\boldsymbol{G}_{\mathrm{c}}(\cdot, \boldsymbol{x}) \in C^{\infty}\left(\Omega ; \mathbb{R}^{3,3}\right)$ and is in particular bounded at $\boldsymbol{\xi}=\boldsymbol{x}$.

The fundamental tensor $\boldsymbol{G}_{\infty}$ and its gradients are positively homogeneous functions: for any $\boldsymbol{r} \in \mathbb{R}^{3} \backslash\{\mathbf{0}\}$ and $\lambda>0$, we have

$$
\boldsymbol{G}_{\infty}(\lambda \boldsymbol{r})=\lambda^{-1} \boldsymbol{G}_{\infty}(\boldsymbol{r}), \quad \boldsymbol{\nabla} \boldsymbol{G}_{\infty}(\lambda \boldsymbol{r})=\lambda^{-2} \nabla \boldsymbol{G}_{\infty}(\boldsymbol{r}), \quad \boldsymbol{\nabla}^{2} \boldsymbol{G}_{\infty}(\boldsymbol{r})=\lambda^{-3} \boldsymbol{\nabla}^{2} \boldsymbol{G}_{\infty}(\lambda \boldsymbol{r}) .
$$

In particular, $\boldsymbol{G}_{\infty}(\boldsymbol{r})$ and $\boldsymbol{\nabla} \boldsymbol{G}_{\infty}(\boldsymbol{r})$, having respectively $O\left(r^{-1}\right)$ and $O\left(r^{-2}\right)$ singularities at the origin, are both $\boldsymbol{L}^{1}(\Omega)$ and $\boldsymbol{L}_{\text {loc }}^{1}\left(\mathbb{R}^{3}\right)$ (tensor-valued) functions. For an isotropic material, $\boldsymbol{G}_{\infty}$ is the well-known Kelvin solution given by:

$$
\boldsymbol{G}_{\infty}(\boldsymbol{r})=\frac{1}{16 \pi \mu(1-\nu) r}[(3-4 \nu) \boldsymbol{I}+\hat{\boldsymbol{r}} \otimes \hat{\boldsymbol{r}}], \text { with } r=|\boldsymbol{r}| \text { and } \hat{\boldsymbol{r}}=\frac{\boldsymbol{r}}{r} .
$$

2.4.2. Integral equation and representation for the perturbation displacement.

On applying the first of equations (2.13) to a test function $\boldsymbol{w} \in \mathcal{V}_{0} \cap C^{1}\left(\omega_{x}\right)$ (where $\omega_{x}$ is a neighbourhood of $\boldsymbol{x}$ ) and applying the first Green's identity to the resulting first term over $\Omega$, the Green's tensor is seen to verify

$$
\langle\boldsymbol{G}(\cdot, \boldsymbol{x}), \boldsymbol{w}\rangle_{\Omega}^{\mathcal{C}}=\boldsymbol{w}(\boldsymbol{x}), \quad \boldsymbol{x} \in \Omega, \quad \forall \boldsymbol{w} \in \mathcal{V}_{0} \cap C^{1}\left(\omega_{x}\right) .
$$

Setting (i) $\boldsymbol{w}=\boldsymbol{G}(\boldsymbol{\xi}, \boldsymbol{x})$ in the variational problem (2.7), (ii) $\boldsymbol{w}=\boldsymbol{v}_{a}$ in (2.17), and (iii) subtracting the two resulting equalities (the integrals arising from steps (i) and (ii) being well-defined for $\boldsymbol{x} \in B_{a} \cup\left(\Omega \backslash \bar{B}_{a}\right)$ due to elliptic interior regularity for $\boldsymbol{v}_{a}$ and the fact that $\boldsymbol{\nabla} \boldsymbol{G}_{\infty}(\cdot-\boldsymbol{x}) \in C^{\infty}(\Omega \backslash\{\boldsymbol{x}\})$ and has an integrable singularity at $\boldsymbol{x}$ ), we obtain the governing volume integro-differential equation (VIE) for $\boldsymbol{v}_{a}$ :

$$
\left(\boldsymbol{I}+\mathcal{L}_{a}\right)\left[\boldsymbol{v}_{a}\right](\boldsymbol{x})=-\mathcal{L}_{a}[\boldsymbol{u}](\boldsymbol{x}), \quad \boldsymbol{x} \in B_{a} \cup\left(\Omega \backslash \bar{B}_{a}\right)
$$

where $\boldsymbol{I}$ is the second-order identity and the linear integro-differential operator $\mathcal{L}_{a}$ is defined by

$$
\mathcal{L}_{a}[\boldsymbol{v}](\boldsymbol{x}):=\langle\boldsymbol{v}, \boldsymbol{G}(\cdot, \boldsymbol{x})\rangle_{B_{a}}^{\Delta \mathcal{C}}=\int_{B_{a}} \boldsymbol{\nabla}_{1} \boldsymbol{G}(\cdot, \boldsymbol{x}): \Delta \mathcal{C}: \boldsymbol{\nabla} \boldsymbol{v} \mathrm{d} V .
$$

In (2.19) and hereinafter, the adopted notational convention for gradient components is such that $(\boldsymbol{\nabla} \boldsymbol{v})_{i k}=$ $v_{i, k}=\partial_{k} v_{i}$ and $(\nabla \boldsymbol{G})_{i j k}=G_{i j, k}=\partial_{k} G_{i j}$. 


\subsection{Free-space transmission problems}

Free-space transmission problems (FSTPs) are auxiliary problems for a perfectly-bonded inhomogeneity $\left(\mathcal{B}, \mathcal{C}^{\star}\right)$ embedded in an infinite elastic medium. On using rescaled coordinates associated with the mapping $B_{a}=\boldsymbol{z}+a \mathcal{B}$, which "sends to infinity" the boundary $\partial \Omega$, asymptotic contributions to $\boldsymbol{v}_{a}$ as $a \rightarrow 0$ will be found to solve FSTPs, making them play a crucial role in the sequel. Accordingly, some known definitions and results pertaining to FSTPs are reviewed in this section for later reference, with useful additional notation introduced in the process, and an auxiliary solvability result for the VIE formulation of a FSTP is given (Prop. 2.2).

\subsubsection{Definition and VIE formulation}

We consider FSTPs defined as follows: given a background displacement field $\boldsymbol{u}$, find the total field $\boldsymbol{u}_{\mathcal{B}}$ such that

$$
\operatorname{div}\left(\mathcal{C}_{\mathcal{B}}: \nabla \boldsymbol{u}_{\mathcal{B}}\right)=\operatorname{div}(\mathcal{C}: \nabla \boldsymbol{u}) \text { in } \mathbb{R}^{3}, \quad \boldsymbol{u}_{\mathcal{B}}(\boldsymbol{\xi})-\boldsymbol{u}(\boldsymbol{\xi})=O\left(|\boldsymbol{\xi}|^{-2}\right) \text { as }|\boldsymbol{\xi}| \rightarrow \infty,
$$

where $\mathcal{C}_{\mathcal{B}}=\mathcal{C}+\Delta \mathcal{C}_{\mathcal{B}}$ with the elasticity perturbation tensor field $\Delta \mathcal{C}_{\mathcal{B}}$ given by $\Delta \mathcal{C}_{\mathcal{B}}=\chi(\mathcal{B}) \Delta \mathcal{C}$. The given decay condition at infinity for the perturbation $\boldsymbol{v}_{\mathcal{B}}:=\boldsymbol{u}_{\mathcal{B}}-\boldsymbol{u}$, which ensures uniqueness of the solution $\boldsymbol{u}_{\mathcal{B}}$, is known from representations of bounded elastostatic fields by volume or layer potentials, while a well-posed variational formulation for problem (2.20) is based on enforcing the decay of $\boldsymbol{v}_{\mathcal{B}}$ by means of a weighted Sobolev space ([16], Chap. XI.B).

By analogy with (2.18), problem (2.20) can then be recast in either of two equivalent VIE forms (a) and (b), depending on whether the main unknown is chosen to be the restriction to $\mathcal{B}$ of $\boldsymbol{u}_{\mathcal{B}}$ (a) or $\boldsymbol{v}_{\mathcal{B}}$ (b):

$$
\text { (a) }\left(\mathcal{I}+\mathcal{L}_{\mathcal{B}}\right)\left[\boldsymbol{u}_{\mathcal{B}}\right](\boldsymbol{x})=\boldsymbol{u}(\boldsymbol{x}), \quad \text { or } \quad\left(\text { b) }\left(\boldsymbol{I}+\mathcal{L}_{\mathcal{B}}\right)\left[\boldsymbol{v}_{\mathcal{B}}\right](\boldsymbol{x})=-\mathcal{L}_{\mathcal{B}}[\boldsymbol{u}](\boldsymbol{x}), \quad \boldsymbol{x} \in \mathcal{B} \cup\left(\mathbb{R}^{3} \backslash \overline{\mathcal{B}}\right),\right.
$$

where the integral operator $\mathcal{L}_{\mathcal{B}}$ is defined, in terms of the full-space Green's tensor $\boldsymbol{G}_{\infty}$ (see (2.15)), by

$$
\mathcal{L}_{\mathcal{B}}[\boldsymbol{v}]:=\int_{\mathcal{B}} \nabla G_{\infty}(\boldsymbol{\xi}-\boldsymbol{x}): \Delta \mathcal{C}: \nabla \boldsymbol{v}(\boldsymbol{\xi}) \mathrm{d} V_{\xi}
$$

Proposition 2.2. Assume that the background and inhomogeneity elasticity tensors $\mathcal{C}$ and $\mathcal{C}^{\star}$ are both positive definite and bounded. The integro-differential operator $\boldsymbol{I}+\mathcal{L}_{\mathcal{B}}: \boldsymbol{H}^{1}(\mathcal{B}) \rightarrow \boldsymbol{H}^{1}(\mathcal{B})$ of the volume integral equation formulation (2.21) of the FSTP is invertible with bounded inverse.

Proposition 2.2 extends Theorem 1 of [22] (which focuses on the solvability of the related integral equation (6.1) for $\boldsymbol{\sigma}^{\star}:=\Delta \mathcal{C}: \varepsilon\left[\boldsymbol{u}_{\mathcal{B}}\right]$, assuming isotropic materials) to general anisotropic materials. Its proof is given in Section 6. We note in particular that the operator $\mathcal{L}_{\mathcal{B}}: \boldsymbol{H}^{1}(\mathcal{B}) \rightarrow \boldsymbol{H}^{1}(\mathcal{B})$ is not compact.

Equations (2.21) are hence well-posed, implying in particular that $\boldsymbol{v}_{\mathcal{B}}=\mathbf{0}$ for any rigid-body background displacement (for which $\mathcal{L}_{\mathcal{B}}[\boldsymbol{u}]=\mathbf{0}$ ). Moreover, we state the following reciprocity identity, whose proof is given in Appendix A, and which will be used later:

Lemma 2.3. Let $\boldsymbol{u}_{\mathcal{B}}, \boldsymbol{u}_{\mathcal{B}}^{\prime}$ solve the FSTP (2.20) with respective background displacements $\boldsymbol{u}, \boldsymbol{u}^{\prime}$. With the notation $\langle\cdot, \cdot\rangle_{\mathcal{B}} \mathcal{C}$ as defined by $(2.5)$, we have:

$$
\left\langle\boldsymbol{u}_{\mathcal{B}}, \boldsymbol{u}^{\prime}\right\rangle_{\mathcal{B}}^{\Delta \mathcal{C}}=\left\langle\boldsymbol{u}_{\mathcal{B}}^{\prime}, \boldsymbol{u}\right\rangle_{\mathcal{B}}^{\Delta \mathcal{C}}
$$

\subsubsection{Polynomial background displacement}

The case where the background displacement $\boldsymbol{u}$ is polynomial plays an important auxiliary role in this study. Any polynomial displacement of degree $n$ may be set in the form

$$
\boldsymbol{u}(\boldsymbol{x})=\boldsymbol{E}_{0}+\sum_{p=1}^{n} \boldsymbol{\varphi}_{p}\left[\boldsymbol{E}_{p}\right](\boldsymbol{x}) \quad \text { with } \quad \boldsymbol{\varphi}_{p}\left[\boldsymbol{E}_{p}\right](\boldsymbol{x}):=\frac{1}{p} \boldsymbol{E}_{p} \cdot \boldsymbol{x}^{\otimes p}
$$


where $\boldsymbol{E}_{p}$ are constant tensors of order $p+1$ that are assumed without loss of generality to be invariant under any permutation of their last $p-1$ indices (as otherwise (2.23) yields the same displacement $\boldsymbol{u}$ upon replacing each $\boldsymbol{E}_{p}$ by its average over all such permutations). In (2.23) and hereinafter, the notation $\boldsymbol{A} \cdot \boldsymbol{B}$ indicates, for any tensors $\boldsymbol{A}, \boldsymbol{B}$ of respective orders $p, q$, their $m$-fold inner product, with $m:=\min (p, q)$ (i.e. the inner product is effected over as many indices as possible), e.g. $(\boldsymbol{A} \bullet \boldsymbol{B})_{i j k}=A_{i j k \ell m n} B_{\ell m n}$ (again with Einstein's summation convention); moreover, the notation $\boldsymbol{x}^{\otimes p}$ is a shorthand for the tensor product $\boldsymbol{x} \otimes \ldots \otimes \boldsymbol{x}$ of order $p$ (e.g. $\left.\boldsymbol{x}^{\otimes 3}=\boldsymbol{x} \otimes \boldsymbol{x} \otimes \boldsymbol{x}\right)$.

The form chosen in (2.23) for the homogeneous polynomials $\boldsymbol{\varphi}_{p}\left[\boldsymbol{E}_{p}\right](\boldsymbol{x})$, together with the assumptions made on $\boldsymbol{E}_{p}$, is such that their strain tensor is $\varepsilon\left[\boldsymbol{\varphi}_{p}\left[\boldsymbol{E}_{p}\right]\right](\boldsymbol{x})=\boldsymbol{E}_{p}^{\mathrm{s}} \boldsymbol{\bullet} \boldsymbol{x}^{\otimes p-1}$, with the superscript "s" indicating symmetrization over the first two indices $\left(e . g . E_{i j k}^{\mathrm{s}}=\left(E_{i j k}+E_{j i k}\right) / 2\right)$. We then denote by $\boldsymbol{U}_{\mathcal{B}}^{(p)}\left[\boldsymbol{E}_{p}\right]$ the solution to the $\operatorname{FSTP}(2.20)$ with given background displacement $\boldsymbol{u}=\boldsymbol{\varphi}_{p}\left[\boldsymbol{E}_{p}\right]$. The displacement perturbation $\boldsymbol{V}_{\mathcal{B}}\left[\boldsymbol{E}_{p}\right]:=\boldsymbol{U}_{\mathcal{B}}\left[\boldsymbol{E}_{p}\right]-\boldsymbol{\varphi}_{p}\left[\boldsymbol{E}_{p}\right]$ then satisfies the integral equation

$$
\left(\boldsymbol{I}+\mathcal{L}_{\mathcal{B}}\right) \boldsymbol{V}_{\mathcal{B}}^{(p)}\left[\boldsymbol{E}_{p}\right]=-\mathcal{L}_{\mathcal{B}}\left[\boldsymbol{\varphi}_{p}\left[\boldsymbol{E}_{p}\right]\right], \text { in } \mathcal{B}
$$

When $\mathcal{B}$ is an ellipsoid, it is well known $[17,18,31]$ that the restriction to $\mathcal{B}$ of $\boldsymbol{U}_{\mathcal{B}}^{(p)}\left[\boldsymbol{E}_{p}\right]$ is polynomial with degree $p$. For the cases $p=1$ and $p=2$, which will be used here, we have (see Appendix B)

$$
\begin{aligned}
& \varepsilon\left(\boldsymbol{U}_{\mathcal{B}}^{(1)}\left[\boldsymbol{E}_{1}\right]\right)(\boldsymbol{x})=\boldsymbol{E}_{1}^{\mathrm{s}}-\mathcal{S}^{1}:\left(\mathcal{C}+\Delta \mathcal{C}: \mathcal{S}^{1}\right)^{-1}: \Delta \mathcal{C}: \boldsymbol{E}_{1}^{\mathrm{s}} \\
& \varepsilon\left(\boldsymbol{U}_{\mathcal{B}}^{(2)}\left[\boldsymbol{E}_{2}\right]\right)(\boldsymbol{x})=\left(\boldsymbol{E}_{2}^{\mathrm{s}}-\mathcal{S}^{2} \cdot\left[\left(\widetilde{\mathcal{C}}+\Delta \mathcal{C}: \mathcal{S}^{2}\right)^{-1} \cdot\left(\widetilde{\Delta \mathcal{C}} \cdot \boldsymbol{E}_{2}^{\mathrm{s}}\right)\right]\right) \cdot \boldsymbol{x}
\end{aligned}
$$

with the sixth-order version $\widetilde{\mathcal{C}}$ of $\mathcal{C}$ defined by $\widetilde{\mathcal{C}}_{i j k \ell m n}=\mathcal{C}_{i j \ell m} \delta_{k n}$ (and similarly for $\widetilde{\Delta \mathcal{C}}$ ), and where $\mathcal{S}^{1}, \mathcal{S}^{2}$ are known constant tensors (respectively of order 4 and 6) depending on $\mathcal{B}$ and $\mathcal{C}$, called Eshelby tensors. The latter are known in closed form if $\mathcal{C}$ is isotropic and in a few other cases [31]; moreover, they depend only on the shape (i.e. aspect ratios) of $\mathcal{B}$, not on its size $\left(\right.$ i.e. $\mathcal{S}^{i}(\lambda \mathcal{B}, \mathcal{C})=\mathcal{S}^{i}(\mathcal{B}, \mathcal{C})$ for $i=1,2$ and any $\left.\lambda>0\right)$. If $\mathcal{B}$ is a ball and $\mathcal{C}$ is isotropic, $\mathcal{S}^{1}$ and $\mathcal{S}^{2}$ are given in component form (with tensors $\mathcal{I}, \mathcal{J}, \mathcal{K}$ as in (2.1)) by [31]

$$
\begin{aligned}
15(1-\nu) \mathcal{S}_{i j k \ell}^{1}= & 5(1+\nu) \mathcal{J}_{i j k \ell}+(8-10 \nu) \mathcal{K}_{i j k \ell} \\
35(1-\nu) \mathcal{S}_{i j k \ell m n}^{2}= & -2 \delta_{i j} \mathcal{I}_{k n \ell m}+(7 \nu-1)\left(\delta_{i j} \delta_{k n}+2 \mathcal{I}_{i j k n}\right) \delta_{\ell m}+2(6-7 \nu) \mathcal{I}_{i j \ell m} \delta_{k n} \\
& +(5-7 \nu)\left(\delta_{i k} \mathcal{I}_{j n \ell m}+\delta_{j k} \mathcal{I}_{i n \ell m}+\delta_{j n} \mathcal{I}_{i k \ell m}+\delta_{i n} \mathcal{I}_{j k \ell m}\right)
\end{aligned}
$$

\subsubsection{Elastic moment tensors}

Elastic moment tensors (EMTs), which are analogs for elasticity of polarization tensors, play a central role in asymptotic expansions involving small inhomogeneities, see e.g. [4,13]. For integers $p, q \geq 1$, let the EMT $\mathcal{A}_{p q}$ be the constant tensor of order $p+q+2$ such that

$$
\left\langle\boldsymbol{U}_{\mathcal{B}}^{(p)}\left[\boldsymbol{E}_{p}\right], \boldsymbol{\varphi}_{q}\left[\boldsymbol{E}_{q}\right]\right\rangle_{\mathcal{B}}^{\Delta \mathcal{C}}=\boldsymbol{E}_{p} \cdot \mathcal{A}_{p q} \bullet \boldsymbol{E}_{q}
$$

for any constant tensors $\boldsymbol{E}_{p}$ and $\boldsymbol{E}_{q}$ of respective orders $p+1$ and $q+1$. Noting that the left-hand side of (2.27) only depends on the partially-symmetrized versions $\boldsymbol{E}_{p}^{\mathrm{s}}, \boldsymbol{E}_{q}^{\mathrm{s}}$ of $\boldsymbol{E}_{p}, \boldsymbol{E}_{q}$ (see Sect. 2.5.1), $\boldsymbol{\mathcal { A }}_{p q}$ is uniquely defined by (2.27) upon enforcing minor symmetries mirroring those of $\boldsymbol{E}_{p}^{\mathrm{s}}, \boldsymbol{E}_{q}^{\mathrm{s}}$, e.g. $\left(\mathcal{A}_{22}\right)_{i j k \ell m n}=\left(\mathcal{A}_{22}\right)_{i j k m \ell n}=$ $\left(\mathcal{A}_{22}\right)_{j i k \ell m n}$.

The practical evaluation of tensors $\mathcal{A}_{p q}$ is made easier by applying the reciprocity identity of Lemma 2.3 to $\left(\boldsymbol{u}_{\mathcal{B}}, \boldsymbol{u}_{\mathcal{B}}^{\prime}\right)=\left(\boldsymbol{U}_{\mathcal{B}}^{(p)}\left[\boldsymbol{E}_{p}\right], \boldsymbol{U}_{\mathcal{B}}^{(q)}\left[\boldsymbol{E}_{q}\right]\right)$ and $\left(\boldsymbol{u}, \boldsymbol{u}^{\prime}\right)=\left(\boldsymbol{\varphi}_{p}\left[\boldsymbol{E}_{p}\right], \boldsymbol{\varphi}_{q}\left[\boldsymbol{E}_{q}\right]\right)$, yielding

$$
\boldsymbol{E}_{p} \cdot \mathcal{A}_{p q} \cdot \boldsymbol{E}_{q}=\boldsymbol{E}_{q} \cdot \mathcal{A}_{q p} \cdot \boldsymbol{E}_{p}
$$

Consequently, knowing the FSTP solution $\boldsymbol{U}_{\mathcal{B}}^{(m)}\left[\boldsymbol{E}_{m}\right]$ with $m:=\min (p, q)$ is enough for evaluating $\mathcal{A}_{p q}$. 
Remark 2.4. The definition of EMTs given in [4], which relies on layer potential representations for $\boldsymbol{U}_{\mathcal{B}}\left[\boldsymbol{E}_{p}\right]$, assumes (i) isotropic background and inhomogeneity materials and (ii) $\operatorname{div}\left(\mathcal{C}: \varepsilon\left[\boldsymbol{\varphi}_{p}\left(\boldsymbol{E}_{p}\right)\right]\right)=\mathbf{0}$. If in addition we also have $\operatorname{div}\left(\mathcal{C}: \varepsilon\left[\boldsymbol{\varphi}_{q}\left(\boldsymbol{E}_{q}\right)\right]\right)=\mathbf{0}$, the present $\mathcal{A}_{p q}$ and its counterpart $\boldsymbol{M}_{\alpha \beta}^{j}$ of [4] (where $\alpha, \beta$ are multi-indices) can be shown to be related through

$$
\boldsymbol{E}_{p} \cdot \mathcal{A}_{p q} \cdot \boldsymbol{E}_{q}=\frac{1}{p q}\left(\boldsymbol{E}_{q}\right)_{\beta \ell} \boldsymbol{M}_{\alpha \beta}^{j} \cdot \boldsymbol{e}_{\ell}\left(\boldsymbol{E}_{p}\right)_{\alpha j} \quad(\text { for }|\alpha|=p,|\beta|=q, \text { and summing over } \alpha, \beta, j, \ell)
$$

\section{Asymptotic Behavior of Perturbation Displacement}

The asymptotic behavior of $\boldsymbol{v}_{a}$ is now investigated, involving the expansion for $a \rightarrow 0$ of integral equation (2.18). It is convenient for this purpose to convert the integrals over the vanishing inhomogeneity $B_{a}$ involved in (2.18) to integrals over the fixed region $\mathcal{B}$. To this aim, points $\boldsymbol{\xi} \in B_{a}$, and the associated differential volume element, are rescaled according to:

$$
\text { (a) } \boldsymbol{\xi}=\boldsymbol{z}+a \overline{\boldsymbol{\xi}}, \quad \text { (b) } \mathrm{d} V_{\xi}=a^{3} \mathrm{~d} \bar{V}_{\bar{\xi}}, \quad \boldsymbol{\xi} \in B_{a}, \overline{\boldsymbol{\xi}} \in \mathcal{B}
$$

Then, we define the isomorphism $\mathcal{P}_{a}: \boldsymbol{H}^{1}\left(B_{a}\right) \rightarrow \boldsymbol{H}^{1}(\mathcal{B})$ associated with this coordinate rescaling, and its inverse $\mathcal{P}_{a}^{-1}$, by

$$
\mathcal{P}_{a}[\boldsymbol{v}](\overline{\boldsymbol{\xi}}):=\boldsymbol{v}(\boldsymbol{z}+a \overline{\boldsymbol{\xi}}) \quad \text { and } \quad \mathcal{P}_{a}^{-1}[\boldsymbol{V}](\boldsymbol{\xi}):=\boldsymbol{V}\left(\frac{\boldsymbol{\xi}-\boldsymbol{z}}{a}\right), \quad \boldsymbol{\xi} \in B_{a}, \overline{\boldsymbol{\xi}} \in \mathcal{B}
$$

The following properties are then verified by $\mathcal{P}_{a}$ :

$$
\text { (a) } \boldsymbol{\nabla}\left(\mathcal{P}_{a}[\boldsymbol{v}]\right)(\overline{\boldsymbol{\xi}})=a \mathcal{P}_{a}[\boldsymbol{\nabla} \boldsymbol{v}](\overline{\boldsymbol{\xi}}), \quad \text { (b) } \boldsymbol{\nabla}\left(\mathcal{P}_{a}^{-1}[\boldsymbol{V}]\right)(\boldsymbol{\xi})=a^{-1} \mathcal{P}_{a}^{-1}[\boldsymbol{\nabla} \boldsymbol{V}](\boldsymbol{\xi}), \quad \boldsymbol{\xi} \in B_{a}, \overline{\boldsymbol{\xi}} \in \mathcal{B}
$$

and

$$
\text { (a) }\|\boldsymbol{v}\|_{\boldsymbol{L}^{2}\left(B_{a}\right)}=a^{3 / 2}\left\|\mathcal{P}_{a}[\boldsymbol{v}]\right\|_{\boldsymbol{L}^{2}(\mathcal{B})}, \quad \text { (b) }\|\boldsymbol{\nabla} \boldsymbol{v}\|_{\boldsymbol{L}^{2}\left(B_{a}\right)}=a^{1 / 2}\left\|\boldsymbol{\nabla}\left(\mathcal{P}_{a}[\boldsymbol{v}]\right)\right\|_{\boldsymbol{L}^{2}(\mathcal{B})} \text {. }
$$

\subsection{Inner expansion}

An inner expansion of $\boldsymbol{v}_{a}(\boldsymbol{x})$ for $\boldsymbol{x} \in B_{a}$ is sought in the form

$$
\boldsymbol{v}_{a}(\boldsymbol{x}) \approx a \boldsymbol{V}_{1}(\overline{\boldsymbol{x}})+a^{2} \boldsymbol{V}_{2}(\overline{\boldsymbol{x}})+a^{3} \boldsymbol{V}_{3}(\overline{\boldsymbol{x}})+a^{4} \boldsymbol{V}_{4}(\overline{\boldsymbol{x}}) \quad\left(\overline{\boldsymbol{x}} \in \mathcal{B}, \boldsymbol{x}=\boldsymbol{z}+a \overline{\boldsymbol{x}} \in B_{a}\right)
$$

in terms of functions $\boldsymbol{V}_{1}, \ldots \boldsymbol{V}_{4}$ of the rescaled coordinates that have to be determined. More precisely, based on the above ansatz, the approximation of $\boldsymbol{v}_{a}(\boldsymbol{x})$ for $\boldsymbol{x} \in B_{a}$ is sought in the form

$$
\boldsymbol{v}_{a}(\boldsymbol{x})=\mathcal{P}_{a}^{-1}\left[\boldsymbol{V}_{a}\right](\boldsymbol{x})+\boldsymbol{\delta}_{a}(\boldsymbol{x}), \quad \text { with } \quad \boldsymbol{V}_{a}:=a \boldsymbol{V}_{1}+a^{2} \boldsymbol{V}_{2}+a^{3} \boldsymbol{V}_{3}+a^{4} \boldsymbol{V}_{4}
$$

where the remainder $\boldsymbol{\delta}_{a}$ is "small", in a sense that will be made precise later (see Prop. 3.1). To derive the corresponding inner expansion of the total displacement field $\boldsymbol{u}_{a}=\boldsymbol{u}+\boldsymbol{v}_{a}$, we note that a Taylor expansion in $a$ yields

$$
\boldsymbol{\nabla} \boldsymbol{u}(\boldsymbol{\xi})=\boldsymbol{g}_{z}+a \boldsymbol{g}_{z}^{(2)} \cdot \overline{\boldsymbol{\xi}}+\frac{a^{2}}{2} \boldsymbol{g}_{z}^{(3)}: \overline{\boldsymbol{\xi}}^{\otimes 2}+\frac{a^{3}}{6} \boldsymbol{g}_{z}^{(4)} \cdot \overline{\boldsymbol{\xi}}^{\otimes 3}+O\left(a^{4}\right)
$$

having introduced for convenience the shorthand notations $\boldsymbol{g}_{z}:=\boldsymbol{\nabla} \boldsymbol{u}(\boldsymbol{z})$ and $\boldsymbol{g}_{z}^{(k)}:=\nabla^{k} \boldsymbol{u}(\boldsymbol{z})$. 


\subsubsection{Determination of the inner expansion}

Aiming at finding governing problems for functions $\boldsymbol{V}_{1}, \ldots \boldsymbol{V}_{4}$, we now proceed with expanding integral equation (2.18) in powers of $a$, by using the rescaled coordinates (3.1a) and the ansatz (3.6) therein. To that end, since $\boldsymbol{G}_{\infty}$ and $\boldsymbol{G}_{\mathrm{c}}$ behave differently in the limit $a \rightarrow 0$, we introduce the decomposition $\mathcal{L}_{a}=\mathcal{L}_{a}^{\infty}+\mathcal{L}_{a}^{\mathrm{c}}$ induced on the integral operator $\mathcal{L}_{a}$ defined by (2.19) by the additive decomposition (2.14) of the Green's tensor, with

$$
\mathcal{L}_{a}^{\infty}[\boldsymbol{v}](\boldsymbol{x}):=\int_{B_{a}} \boldsymbol{\nabla} \boldsymbol{G}_{\infty}(\cdot-\boldsymbol{x}): \Delta \mathcal{C}: \nabla \boldsymbol{v} \mathrm{d} V, \quad \mathcal{L}_{a}^{\mathrm{c}}[\boldsymbol{v}](\boldsymbol{x}):=\int_{B_{a}} \boldsymbol{\nabla}_{1} \boldsymbol{G}_{\mathrm{C}}(\cdot, \boldsymbol{x}): \Delta \mathcal{C}: \boldsymbol{\nabla} \boldsymbol{v} \mathrm{d} V
$$

Equation (2.18) then becomes

$$
\left(\boldsymbol{I}+\mathcal{L}_{a}^{\infty}\right)\left[\boldsymbol{v}_{a}\right](\boldsymbol{x})=-\mathcal{L}_{a}^{\infty}[\boldsymbol{u}](\boldsymbol{x})-\mathcal{L}_{a}^{\mathrm{c}}\left[\boldsymbol{u}+\boldsymbol{v}_{a}\right](\boldsymbol{x}), \quad \forall \boldsymbol{x} \in B_{a} \cup \Omega \backslash \bar{B}_{a}
$$

We first note that the homogeneity property (2.16) implies

$$
\nabla \boldsymbol{G}_{\infty}(\boldsymbol{\xi}-\boldsymbol{x})=a^{-2} \nabla \boldsymbol{G}_{\infty}(\overline{\boldsymbol{\xi}}-\overline{\boldsymbol{x}}) .
$$

Using this, together with rescaled coordinates (3.1a) and its consequences (3.1b) and (3.3a), in $\mathcal{L}_{a}^{\infty}$, we find that

$$
\mathcal{L}_{a}^{\infty}[\boldsymbol{w}](\boldsymbol{x})=\mathcal{P}_{a}^{-1} \mathcal{L}_{\mathcal{B}} \mathcal{P}_{a}[\boldsymbol{w}](\boldsymbol{x})
$$

for any $\boldsymbol{w} \in \boldsymbol{H}^{1}\left(B_{a}\right)$ and $\boldsymbol{x} \in B_{a} \cup\left(\mathbb{R}^{3} \backslash \bar{B}_{a}\right)$, with the integral operator $\mathcal{L}_{\mathcal{B}}$ defined by (2.22). Then, evaluating $\mathcal{L}_{a}^{\infty}\left[\boldsymbol{v}_{a}\right]$ and $\mathcal{L}_{a}^{\infty}[\boldsymbol{u}]$ using (3.6), (3.7) and the above rescaling property (3.10), we obtain

$$
\begin{aligned}
\mathcal{P}_{a} \mathcal{L}_{a}^{\infty}\left[\boldsymbol{v}_{a}\right](\overline{\boldsymbol{x}}) & =\mathcal{L}_{\mathcal{B}}\left[a \boldsymbol{V}_{1}+a^{2} \boldsymbol{V}_{2}+a^{3} \boldsymbol{V}_{3}+a^{4} \boldsymbol{V}_{4}\right](\overline{\boldsymbol{x}})+\mathcal{P}_{a} \mathcal{L}_{a}^{\infty}\left[\boldsymbol{\delta}_{a}\right](\overline{\boldsymbol{x}}) \\
\mathcal{P}_{a} \mathcal{L}_{a}^{\infty}[\boldsymbol{u}](\overline{\boldsymbol{x}}) & =\mathcal{L}_{\mathcal{B}}\left[a \boldsymbol{\varphi}_{1}\left[\boldsymbol{g}_{z}\right]+a^{2} \boldsymbol{\varphi}_{2}\left[\boldsymbol{g}_{z}^{(2)}\right]+\frac{1}{2} a^{3} \boldsymbol{\varphi}_{3}\left[\boldsymbol{g}_{z}^{(3)}\right]+\frac{1}{6} a^{4} \boldsymbol{\varphi}_{4}\left[\boldsymbol{g}_{z}^{(4)}\right]\right](\overline{\boldsymbol{x}})+o\left(a^{4}\right)
\end{aligned}
$$

having expressed the Taylor expansion (3.7) in terms of the vector-valued homogeneous polynomials $\boldsymbol{\varphi}_{m}$ introduced in (2.23).

To expand $\mathcal{L}_{a}^{\mathrm{c}}\left[\boldsymbol{v}_{a}\right]$ and $\mathcal{L}_{a}^{\mathrm{c}}[\boldsymbol{u}]$, we note that $\boldsymbol{\nabla}_{1} \boldsymbol{G}_{\mathrm{c}}$, being a smooth function in $\Omega \times \Omega$, has the Taylor expansion

$$
\boldsymbol{\nabla}_{1} \boldsymbol{G}(\boldsymbol{\xi}, \boldsymbol{x})=\boldsymbol{\nabla}_{1} \boldsymbol{G}_{\mathrm{c}}(\boldsymbol{z}, \boldsymbol{z})+a\left(\boldsymbol{\nabla}_{11} \boldsymbol{G}_{\mathrm{c}}(\boldsymbol{z}, \boldsymbol{z}) \cdot \overline{\boldsymbol{\xi}}+\boldsymbol{\nabla}_{12} \boldsymbol{G}_{\mathrm{c}}(\boldsymbol{z}, \boldsymbol{z}) \cdot \overline{\boldsymbol{x}}\right)+o(a),
$$

where rescaled coordinates (3.1a) have been introduced and notations $\nabla_{1}$ etc. are as defined after (2.9). We then obtain

$$
\mathcal{P}_{a} \mathcal{L}_{a}^{c}\left[\boldsymbol{u}+\boldsymbol{v}_{a}\right](\overline{\boldsymbol{x}})=a^{3} \boldsymbol{f}_{z}^{(3)}+a^{4}\left[\boldsymbol{f}_{z}^{(4)}+\boldsymbol{L}_{z} \cdot \overline{\boldsymbol{x}}\right]+o\left(a^{4}\right)
$$

with the constant vectors $\boldsymbol{f}_{z}^{(3)}, \boldsymbol{f}_{z}^{(4)}$ and the constant tensor $\boldsymbol{L}_{z}$ given by

$$
\begin{aligned}
\boldsymbol{f}_{z}^{(3)} & =\int_{\mathcal{B}} \nabla_{1} \boldsymbol{G}_{\mathrm{c}}(\boldsymbol{z}, \boldsymbol{z}): \Delta \mathcal{C}:\left(\boldsymbol{g}_{z}+\nabla \boldsymbol{V}_{1}(\overline{\boldsymbol{\xi}})\right) \mathrm{d} \bar{V}_{\bar{\xi}} \\
\boldsymbol{f}_{z}^{(4)} & =\int_{\mathcal{B}} \nabla_{1} \boldsymbol{G}_{\mathrm{c}}(\boldsymbol{z}, \boldsymbol{z}): \Delta \mathcal{C}:\left(\boldsymbol{g}_{z}^{(2)} \cdot \overline{\boldsymbol{\xi}}+\nabla \boldsymbol{V}_{2}(\overline{\boldsymbol{\xi}})\right) \mathrm{d} \bar{V}_{\bar{\xi}}+\int_{\mathcal{B}}\left(\nabla_{11} \boldsymbol{G}_{\mathrm{c}}(\boldsymbol{z}, \boldsymbol{z}) \cdot \overline{\boldsymbol{\xi}}\right): \Delta \mathcal{C}:\left(\boldsymbol{g}_{z}+\nabla \boldsymbol{V}_{1}(\overline{\boldsymbol{\xi}})\right) \mathrm{d} \bar{V}_{\bar{\xi}} \\
\boldsymbol{L}_{z} \cdot \overline{\boldsymbol{x}} & =\int_{\mathcal{B}}\left(\nabla_{12} \boldsymbol{G}_{\mathrm{c}}(\boldsymbol{z}, \boldsymbol{z}) \cdot \overline{\boldsymbol{x}}\right): \Delta \mathcal{C}:\left(\boldsymbol{g}_{z}+\nabla \boldsymbol{V}_{1}(\overline{\boldsymbol{\xi}})\right) \mathrm{d} \bar{V}_{\overline{\boldsymbol{\xi}}}
\end{aligned}
$$


The desired equations for $\boldsymbol{V}_{1}, \ldots \boldsymbol{V}_{4}$ then result from setting to zero the $O(a)$ to $O\left(a^{4}\right)$ terms arising in (3.9), with the help of $(3.11 \mathrm{a}, \mathrm{b})$ and $(3.12)$. First, the $O(a)$ and $O\left(a^{2}\right)$ equations are

$$
\begin{array}{llrl}
\left(\boldsymbol{I}+\mathcal{L}_{\mathcal{B}}\right)\left[\boldsymbol{V}_{1}\right](\overline{\boldsymbol{x}}) & =-\mathcal{L}_{\mathcal{B}}\left[\boldsymbol{\varphi}_{1}\left[\boldsymbol{g}_{z}\right]\right](\overline{\boldsymbol{x}}), & & \overline{\boldsymbol{x}} \in \mathcal{B}, \\
\left(\boldsymbol{I}+\mathcal{L}_{\mathcal{B}}\right)\left[\boldsymbol{V}_{2}\right](\overline{\boldsymbol{x}})=-\mathcal{L}_{\mathcal{B}}\left[\boldsymbol{\varphi}_{2}\left[\boldsymbol{g}_{z}^{(2)}\right]\right](\overline{\boldsymbol{x}}), & & \overline{\boldsymbol{x}} \in \mathcal{B} .
\end{array}
$$

They are seen to correspond to the FSTPs (2.24) with polynomial background displacements $\boldsymbol{\varphi}_{1}\left[\boldsymbol{g}_{z}\right]$ and $\boldsymbol{\varphi}_{2}\left[\boldsymbol{g}_{z}^{(2)}\right]$, respectively, so that $\boldsymbol{V}_{1}$ and $\boldsymbol{V}_{2}$ are well defined; they are given by

$$
\boldsymbol{V}_{1}=\boldsymbol{V}_{\mathcal{B}}^{(1)}\left[\boldsymbol{g}_{z}\right], \quad \boldsymbol{V}_{2}=\boldsymbol{V}_{\mathcal{B}}^{(2)}\left[\boldsymbol{g}_{z}^{(2)}\right] .
$$

Then, the $O\left(a^{3}\right)$ and $O\left(a^{4}\right)$ equations are found to be

$$
\begin{array}{ll}
\left(\boldsymbol{I}+\mathcal{L}_{\mathcal{B}}\right)\left[\boldsymbol{V}_{3}\right](\overline{\boldsymbol{x}})=-\frac{1}{2} \mathcal{L}_{\mathcal{B}}\left[\boldsymbol{\varphi}_{3}\left[\boldsymbol{g}_{z}^{(3)}\right]\right](\overline{\boldsymbol{x}})-\boldsymbol{f}_{z}^{(3)}, & \overline{\boldsymbol{x}} \in \mathcal{B}, \\
\left(\boldsymbol{I}+\mathcal{L}_{\mathcal{B}}\right)\left[\boldsymbol{V}_{4}\right](\overline{\boldsymbol{x}})=-\frac{1}{6} \mathcal{L}_{\mathcal{B}}\left[\boldsymbol{\varphi}_{4}\left[\boldsymbol{g}_{z}^{(4)}\right]\right](\overline{\boldsymbol{x}})-\boldsymbol{f}_{z}^{(4)}-\boldsymbol{L}_{z} \cdot \overline{\boldsymbol{x}}, & \overline{\boldsymbol{x}} \in \mathcal{B},
\end{array}
$$

where $\boldsymbol{f}_{z}^{(3)}, \boldsymbol{f}_{z}^{(4)}, \boldsymbol{L}_{z}$, given by (3.12), depend on $\boldsymbol{V}_{1}$ and $\boldsymbol{V}_{2}$ and can be given a more compact form by using (3.14) and the elastic moment tensors defined by (2.27), to obtain

$$
\begin{aligned}
\boldsymbol{f}_{z}^{(3)} & =\nabla_{1} \boldsymbol{G}_{\mathrm{c}}(\boldsymbol{z}, \boldsymbol{z}): \mathcal{A}_{11}: \boldsymbol{g}_{z}, \\
\boldsymbol{f}_{z}^{(4)} & =\left(\nabla_{1} \boldsymbol{G}_{\mathrm{c}}(\boldsymbol{z}, \boldsymbol{z}): \mathcal{A}_{12}\right) \cdot \boldsymbol{g}_{z}^{(2)}+\nabla_{11} \boldsymbol{G}_{\mathrm{c}}(\boldsymbol{z}, \boldsymbol{z}) \cdot\left(\mathcal{A}_{21}: \boldsymbol{g}_{z}\right), \\
\boldsymbol{L}_{z} \cdot \overline{\boldsymbol{x}} & =\left(\nabla_{12} \boldsymbol{G}_{\mathrm{c}}(\boldsymbol{z}, \boldsymbol{z}) \cdot \overline{\boldsymbol{x}}\right): \mathcal{A}_{11}: \boldsymbol{g}_{z}
\end{aligned}
$$

Equations $(3.15 \mathrm{a}, \mathrm{b})$ also correspond to FSTPs with polynomial background displacements, and we find

$$
\begin{aligned}
& \boldsymbol{V}_{3}=\frac{1}{2} \boldsymbol{V}_{\mathcal{B}}^{(3)}\left[\boldsymbol{g}_{z}^{(3)}\right]-\boldsymbol{f}_{z}^{(3)}, \\
& \boldsymbol{V}_{4}=\frac{1}{6} \boldsymbol{V}_{\mathcal{B}}^{(4)}\left[\boldsymbol{g}_{z}^{(4)}\right]-\boldsymbol{U}_{\mathcal{B}}^{(1)}\left[\boldsymbol{L}_{z}\right](\overline{\boldsymbol{x}})-\boldsymbol{f}_{z}^{(4)} .
\end{aligned}
$$

Finally, using (3.14) and (3.16) in (3.6), the proposed inner approximation $\boldsymbol{V}_{a}$ of $\boldsymbol{v}_{a}$ is completely specified.

\subsubsection{Justification of the inner expansion}

To justify the order of approximation between the exact displacement perturbation $\boldsymbol{v}_{a}$ and its above-defined inner expansion $\boldsymbol{V}_{a}$, we need to determine the asymptotic behavior of the inner approximation error $\boldsymbol{\delta}_{a}:=\boldsymbol{v}_{a}-$ $\mathcal{P}_{a}^{-1}\left[\boldsymbol{V}_{a}\right]$ as $a \rightarrow 0$. To this aim, the inner approximation order and error are now evaluated in terms of $\boldsymbol{H}^{1}\left(B_{a}\right)$ norms, as this will provide the relevant estimates for subsequent estimation of the cost functional expansion error (Sect. 4). We note that the highest-order term $\boldsymbol{v}_{4}:=\mathcal{P}_{a}^{-1}\left[a^{4} \boldsymbol{V}_{4}\right]$ in the inner approximation of $\boldsymbol{v}_{a}$ is, by virtue of (3.4a,b), such that $\left\|\boldsymbol{v}_{4}\right\|_{\boldsymbol{L}^{2}\left(B_{a}\right)}=a^{11 / 2}\left\|\boldsymbol{V}_{4}\right\|_{\boldsymbol{L}^{2}(\mathcal{B})}=O\left(a^{11 / 2}\right)$ and $\left\|\boldsymbol{\nabla} \boldsymbol{v}_{4}\right\|_{\boldsymbol{L}^{2}\left(B_{a}\right)}=a^{9 / 2}\left\|\boldsymbol{\nabla} \boldsymbol{V}_{4}\right\|_{\boldsymbol{L}^{2}(\mathcal{B})}=O\left(a^{9 / 2}\right)$, so that $\left\|\boldsymbol{v}_{4}\right\|_{\boldsymbol{H}^{1}\left(B_{a}\right)}=O\left(a^{9 / 2}\right)$. The justification of the inner approximation (3.6), (3.14), (3.16) then rests on showing that $\left\|\boldsymbol{\delta}_{a}\right\|_{\boldsymbol{H}^{1}\left(B_{a}\right)}=o\left(a^{9 / 2}\right)$, which the following estimate achieves:

Proposition 3.1 (Error estimate for the inner approximation of displacement). Assume that the background and inhomogeneity elasticity tensors $\mathcal{C}$ and $\mathcal{C}^{\star}$ are both positive definite and bounded. There exists $a_{1}>0$ and a constant $C>0$ independent of a such that

$$
\left\|\boldsymbol{\delta}_{a}\right\|_{\boldsymbol{H}^{1}\left(B_{a}\right)} \leq C a^{11 / 2} \quad \text { for all } a<a_{1} .
$$


Proof. Setting $\boldsymbol{v}_{a}=\mathcal{P}_{a}^{-1} \boldsymbol{V}_{a}+\boldsymbol{\delta}_{a}$ in integral equation (2.18) according to definition (3.6) of the inner approximation, the expansion error $\boldsymbol{\delta}_{a}$ is found to satisfy an integral equation of the form $\left(\boldsymbol{I}+\mathcal{L}_{a}\right)\left[\boldsymbol{\delta}_{a}\right](\boldsymbol{x})=\boldsymbol{\gamma}_{a}(\boldsymbol{x})$. The proof of estimate (3.18), whose details (together with the precise expression of $\gamma_{a}$ ) are deferred to Section 7, then consists in (i) proving that the inverse operator $\left(\boldsymbol{I}+\mathcal{L}_{a}\right)^{-1}: \boldsymbol{H}^{1}\left(B_{a}\right) \rightarrow \boldsymbol{H}^{1}\left(B_{a}\right)$ exists and is bounded independently of $a$ for any small enough $a$ and (ii) estimating $\left\|\gamma_{a}\right\|_{\boldsymbol{H}^{1}\left(\left(B_{a}\right)\right.}$. The assumptions made on $\mathcal{C}$ and $\mathcal{C}^{\star}$ guarantee the bounded invertibility of $\mathcal{L}_{\mathcal{B}}$ (see Prop. 2.2), which is used in the proof given in Section 7.

\subsection{Outer expansion}

We now turn to the expansion of $\boldsymbol{v}_{a}(\boldsymbol{x})$ for $\boldsymbol{x} \in \Omega, \boldsymbol{x} \neq \boldsymbol{z}$ (outer expansion), assuming $a$ small enough to have $\boldsymbol{x} \notin B_{a}$. In this case, $\boldsymbol{v}_{a}(\boldsymbol{x})$ is given by (2.18) used as integral representation:

$$
\boldsymbol{v}_{a}(\boldsymbol{x})=-\mathcal{L}_{a}\left[\boldsymbol{u}_{a}\right](\boldsymbol{x}) \quad \boldsymbol{x} \in \Omega \backslash \bar{B}_{a} .
$$

Since $\boldsymbol{x} \notin B_{a}, \boldsymbol{\xi} \mapsto \boldsymbol{G}(\boldsymbol{\xi}, \boldsymbol{x})$ is smooth for $\boldsymbol{\xi} \in B_{a}$, and one therefore has $\boldsymbol{\nabla}_{1} \boldsymbol{G}(\boldsymbol{\xi}, \boldsymbol{x})=\boldsymbol{\nabla}_{1} \boldsymbol{G}(\boldsymbol{z}, \boldsymbol{x})+O(a)$; moreover, the inner expansion (3.5) truncated to leading order gives $\boldsymbol{\nabla} \boldsymbol{u}_{a}(\boldsymbol{\xi})=\boldsymbol{g}_{z}+\mathcal{P}_{a}^{-1}\left[\boldsymbol{\nabla} \boldsymbol{V}_{1}\right](\boldsymbol{\xi})+O(a)$. Using this, together with the rescaling (3.1), in (3.19) yields the leading-order outer expansion

$$
\boldsymbol{v}_{a}(\boldsymbol{x})=a^{3} \boldsymbol{v}_{\text {out }}(\boldsymbol{x} ; \boldsymbol{z})+O\left(a^{4}\right), \quad \text { with } \quad \boldsymbol{v}_{\text {out }}(\boldsymbol{x} ; \boldsymbol{z}):=-\boldsymbol{\nabla}_{1} \boldsymbol{G}(\boldsymbol{z}, \boldsymbol{x}): \mathcal{A}_{11}: \boldsymbol{g}_{z} \quad(\boldsymbol{x} \in \Omega, \boldsymbol{x} \neq \boldsymbol{z}),
$$

which holds pointwise and is known from many previous studies, e.g. [5]. Expansion (3.20) also holds pointwise on $\partial \Omega$ if $\partial \Omega$ is $C^{1,1}$ (which ensures continuity up to $\partial \Omega$ of $\boldsymbol{x} \mapsto \boldsymbol{G}(\boldsymbol{z}, \boldsymbol{x})=\boldsymbol{G}^{\mathrm{T}}(\boldsymbol{x}, \boldsymbol{z})$ by e.g. [29], Thm. 4.18). Such additional smoothness requirement on $\partial \Omega$ is avoided by the following version of the outer expansion:

Lemma 3.2. Let $D \Subset \Omega$ be an open subset of $\Omega$ such that $\boldsymbol{z} \in D$, and assume a is small enough to have $\operatorname{dist}\left(B_{a}, \partial D\right)>0$. Then: $\left\|\boldsymbol{v}_{a}-a^{3} \boldsymbol{v}_{\text {out }}\right\|_{\boldsymbol{H}^{1}(\Omega \backslash \bar{D})}=O\left(a^{4}\right)$.

Proof. Let $D^{\prime} \Subset D$ be an open subset of $D$ such that $B_{a} \Subset D^{\prime}$, and define $\boldsymbol{w}:=\left(\boldsymbol{v}_{a}-a^{3} \boldsymbol{v}_{\text {out }}\right) \theta$ in $\Omega$ (with $\theta$ a smooth cut-off function, independent of $a$, equal to 1 in $\Omega \backslash D$ and vanishing in $\left.D^{\prime}\right)$. Let $\boldsymbol{b}:=-\operatorname{div}(\mathcal{C}: \varepsilon[\boldsymbol{w}])$. Then, since $\operatorname{div}\left(\mathcal{C}: \varepsilon\left[\boldsymbol{v}_{a}\right]\right)=\operatorname{div}\left(\mathcal{C}: \varepsilon\left[\boldsymbol{v}_{\text {out }}\right]\right)=\mathbf{0}$ in $\Omega-\bar{D}$, we have $\operatorname{supp}(\boldsymbol{b}) \subset \overline{D \backslash D^{\prime}}$. Expansion (3.20) hold pointwise in $\overline{D \backslash D^{\prime}}$, and so does the corresponding expansion of $\boldsymbol{\nabla} \boldsymbol{v}_{a}$ by virtue of $\boldsymbol{\xi} \mapsto \boldsymbol{\nabla}_{2} \boldsymbol{G}(\boldsymbol{\xi}, \boldsymbol{x})$ being smooth for $\boldsymbol{\xi} \in B_{a}$, implying that $\|\boldsymbol{b}\|_{\boldsymbol{L}^{2}(\Omega)}=\|\boldsymbol{b}\|_{\boldsymbol{L}^{2}\left(D \backslash D^{\prime}\right)}=O\left(a^{4}\right)$. The Lemma therefore follows from the Lax-Milgram theorem applied to the problem $-\operatorname{div}(\mathcal{C}: \varepsilon[\boldsymbol{w}])=\boldsymbol{b}$ with boundary conditions $\boldsymbol{w}=\mathbf{0}$ on $\Gamma_{\mathrm{D}}, \boldsymbol{t}[\boldsymbol{w}]=\mathbf{0}$ on $\Gamma_{\mathrm{N}}$.

\section{Misfit FUnCtion EXPANSION}

Exploiting and collecting the results established thus far, we are now in a position to formulate and justify the $O\left(a^{6}\right)$ expansion of $\mathbb{J}\left(\mathcal{C}_{a}\right)$ for a single inhomogeneity (see Sect. 5.3 for the case of multiple inhomogeneities with fixed locations). The most general form of the proposed expansion, valid for a small inhomogeneity of arbitrary shape, is first given in Theorem 4.1, which is the main result of this work. Then, this result is specialized to the sub-class of centrally-symmetric inclusions (Sect. 4.2), which includes the important special cases of ellipsoidal and spherical inhomogeneities for which explicit forms of the expansion coefficients can be given (Sect. 4.3).

\subsection{Inhomogeneity of arbitrary shape}

Theorem 4.1. For a single inhomogeneity, characterized by its geometrical support $B_{a}:=\boldsymbol{z}+$ aB and elasticity tensor $\mathcal{C}^{\star}$ and embedded in the three-dimensional reference medium $\Omega$ with elasticity tensor $\mathcal{C}$, the $O\left(a^{6}\right)$ expansion of any objective function $\mathbb{J}\left(\mathcal{C}_{a}\right)$ of format $(2.8)$ with densities $\Psi_{\Omega}(\boldsymbol{x}, \boldsymbol{w}), \Psi_{\Gamma}(\boldsymbol{x}, \boldsymbol{w})$ that are twice differentiable in their second argument with second-order derivatives having $C^{0, \gamma}$ regularity for some $\gamma>0$ is

$$
\mathbb{J}\left(\mathcal{C}_{a}\right)=\mathbb{J}(\mathcal{C})+a^{3} \mathcal{T}_{3}(\boldsymbol{z})+a^{4} \mathcal{T}_{4}(\boldsymbol{z})+a^{5} \mathcal{T}_{5}(\boldsymbol{z})+a^{6} \mathcal{T}_{6}(\boldsymbol{z})+o\left(a^{6}\right)
$$


with the topological derivatives $\mathcal{T}_{3}, \ldots \mathcal{T}_{6}$ given by

$$
\begin{aligned}
\mathcal{T}_{3}(\boldsymbol{z})= & -\boldsymbol{g}_{z}: \mathcal{A}_{11}: \hat{\boldsymbol{g}}_{z} \\
\mathcal{T}_{4}(\boldsymbol{z})= & -\boldsymbol{g}_{z}: \mathcal{A}_{12} \bullet \hat{\boldsymbol{g}}_{z}^{(2)}-\hat{\boldsymbol{g}}_{z}: \mathcal{A}_{12} \bullet \boldsymbol{g}_{z}^{(2)} \\
\mathcal{T}_{5}(\boldsymbol{z})= & -\frac{1}{2} \boldsymbol{g}_{z}: \mathcal{A}_{13} \bullet \hat{\boldsymbol{g}}_{z}^{(3)}-\boldsymbol{g}_{z}^{(2)} \bullet \mathcal{A}_{22} \bullet \hat{\boldsymbol{g}}_{z}^{(2)}-\frac{1}{2} \hat{\boldsymbol{g}}_{z}: \mathcal{A}_{13} \bullet \boldsymbol{g}_{z}^{(3)} \\
\mathcal{T}_{6}(\boldsymbol{z})= & -\frac{1}{6} \boldsymbol{g}_{z}: \mathcal{A}_{14} \bullet \hat{\boldsymbol{g}}_{z}^{(4)}-\frac{1}{2} \boldsymbol{g}_{z}^{(2)} \bullet \mathcal{A}_{23} \bullet \hat{\boldsymbol{g}}_{z}^{(3)}-\frac{1}{2} \hat{\boldsymbol{g}}_{z}^{(2)} \bullet \mathcal{A}_{23} \bullet \boldsymbol{g}_{z}^{(3)}-\frac{1}{6} \hat{\boldsymbol{g}}_{z}: \mathcal{A}_{14} \bullet \boldsymbol{g}_{z}^{(4)} \\
& \quad+\boldsymbol{g}_{z}: \mathcal{A}_{11}: \nabla_{12} \boldsymbol{G}_{c}(\boldsymbol{z}, \boldsymbol{z}): \mathcal{A}_{11}: \hat{\boldsymbol{g}}_{z}+\frac{1}{2} J^{\prime \prime}\left(\boldsymbol{u} ; \boldsymbol{v}_{\text {out }}\right)
\end{aligned}
$$

In the above formulas, the elastic moment tensors $\mathcal{A}_{p q}(p=1,2, q=1,2,3,4)$ are defined by $(2.27)$ in terms of solutions $\boldsymbol{U}_{\mathcal{B}}^{(p)}$ to free-space transmission problems with polynomial background displacement of degree $p$ (see Sect. 2.5.2), the function $\boldsymbol{v}_{\text {out }}$ is given by (3.20), and $\boldsymbol{G}_{c}$ is the complementary Green's tensor introduced in $(2.14)$.

Proof. The proof proceeds by separately expanding in powers of $a$ each term of expansion (2.9) of $\mathbb{J}\left(\mathcal{C}_{a}\right)$. For the first term, using (2.11), we have

$$
J^{\prime}\left(\boldsymbol{u} ; \boldsymbol{v}_{a}\right)=\left\langle\hat{\boldsymbol{u}}, \boldsymbol{u}_{a}\right\rangle_{B_{a}}^{\Delta \mathcal{C}}=\left\langle\hat{\boldsymbol{u}}, \boldsymbol{u}+\mathcal{P}_{a}^{-1}\left[\boldsymbol{V}_{a}\right]\right\rangle_{B_{a}}^{\Delta \mathcal{C}}+\left\langle\hat{\boldsymbol{u}}, \boldsymbol{\delta}_{a}\right\rangle_{B_{a}}^{\Delta \mathcal{C}}
$$

with $\mathcal{P}_{a}$ as defined by (3.2). The first term in the right-hand side of (4.2a) is first expanded to order $O\left(a^{6}\right)$ by (i) expressing the integral using the rescaling (3.1) for the coordinate $\boldsymbol{\xi}$ and the differential element $\mathrm{d} V_{\boldsymbol{\xi}}$ (see (3.1)), (ii) using definition (3.6) of $\boldsymbol{V}_{a}$, expansion (3.7) for $\mathcal{P}_{a}[\boldsymbol{\nabla} \boldsymbol{u}]$ and the corresponding expansion for $\mathcal{P}_{a}[\boldsymbol{\nabla} \hat{\boldsymbol{u}}]$, (iii) using expressions (3.14) and (3.16) for $\boldsymbol{V}_{1}, \ldots, \boldsymbol{V}_{4}$, to obtain

$$
\begin{aligned}
&\left\langle\hat{\boldsymbol{u}}, \boldsymbol{u}+\mathcal{P}_{a}^{-1}\left[\boldsymbol{V}_{a}\right]\right\rangle_{B_{a}}^{\Delta \mathcal{C}} \\
&=a^{3} \int_{\mathcal{B}}\left[\boldsymbol{\nabla} \boldsymbol{U}_{\mathcal{B}}^{(1)}\left[\boldsymbol{g}_{z}^{(1)}\right]+a \boldsymbol{\nabla} \boldsymbol{U}_{\mathcal{B}}^{(2)}\left[\boldsymbol{g}_{z}^{(2)}\right]+\frac{a^{2}}{2} \nabla \boldsymbol{U}_{\mathcal{B}}^{(3)}\left[\boldsymbol{g}_{z}^{(3)}\right]+a^{3}\left(\frac{1}{6} \boldsymbol{\nabla} \boldsymbol{U}_{\mathcal{B}}^{(4)}\left[\boldsymbol{g}_{z}^{(4)}\right]-\nabla \boldsymbol{U}_{\mathcal{B}}^{(1)}\left[\boldsymbol{L}_{z}\right]\right)\right] \\
&: \Delta \mathcal{C}:\left[\hat{\boldsymbol{g}}_{z}+a \hat{\boldsymbol{g}}_{z}^{(2)} \cdot \overline{\boldsymbol{\xi}}+\frac{a^{2}}{2} \hat{\boldsymbol{g}}_{z}^{(3)}: \overline{\boldsymbol{\xi}}^{\otimes 2}+\frac{a^{3}}{6} \hat{\boldsymbol{g}}_{z}^{(4)} \bullet \overline{\boldsymbol{\xi}}^{\otimes 3}\right] \mathrm{d} \bar{V}_{\bar{\xi}}+o\left(a^{6}\right)
\end{aligned}
$$

(where we used the fact that in (3.16) the last terms of $\boldsymbol{V}_{3}$ and $\boldsymbol{V}_{4}$ are constant in $\overline{\boldsymbol{\xi}}$ ). Expanding the inner product of sums into a sum of inner products, retaining only contributions of order at most $O\left(a^{6}\right)$ and noting that each resulting summand has the form (2.27), we obtain

$$
\begin{aligned}
\left\langle\hat{\boldsymbol{u}}, \boldsymbol{u}_{a}\right\rangle_{B_{a}}^{\Delta \mathcal{C}}= & \left.a^{3}\left(\boldsymbol{g}_{z}: \mathcal{A}_{11}: \hat{\boldsymbol{g}}_{z}\right)+a^{4}-\boldsymbol{g}_{z}: \mathcal{A}_{12} \bullet \hat{\boldsymbol{g}}_{z}^{(2)}+\hat{\boldsymbol{g}}_{z}: \mathcal{A}_{12} \bullet \boldsymbol{g}_{z}^{(2)}\right) \\
& +a^{5}\left(\frac{1}{2} \boldsymbol{g}_{z}: \mathcal{A}_{13} \bullet \hat{\boldsymbol{g}}_{z}^{(3)}+\boldsymbol{g}_{z}^{(2)} \bullet \mathcal{A}_{22} \bullet \hat{\boldsymbol{g}}_{z}^{(2)}+\frac{1}{2} \hat{\boldsymbol{g}}_{z}: \mathcal{A}_{13} \bullet \boldsymbol{g}_{z}^{(3)}\right) \\
& +a^{6}\left(\frac{1}{6} \hat{\boldsymbol{g}}_{z}: \mathcal{A}_{14} \bullet \boldsymbol{g}_{z}^{(4)}+\frac{1}{2} \hat{\boldsymbol{g}}_{z}^{(2)} \bullet \mathcal{A}_{23} \bullet \boldsymbol{g}_{z}^{(3)}+\frac{1}{2} \boldsymbol{g}_{z}^{(2)} \bullet \mathcal{A}_{23} \bullet \hat{\boldsymbol{g}}_{z}^{(3)}+\frac{1}{6} \boldsymbol{g}_{z}: \mathcal{A}_{14} \bullet \hat{\boldsymbol{g}}_{z}^{(4)}\right. \\
& \left.-\boldsymbol{g}_{z}: \mathcal{A}_{11}: \nabla_{12} \boldsymbol{G}_{\mathrm{c}}(\boldsymbol{z}, \boldsymbol{z}): \mathcal{A}_{11}: \hat{\boldsymbol{g}}_{z}\right)+o\left(a^{6}\right),
\end{aligned}
$$

where the reciprocity relations (2.28) for the elastic moment tensors $\mathcal{A}_{p q}$ have also be used wherever appropriate. Moreover, the second term in the right-hand side of (4.2a) admits the following estimate by virtue of Proposition 3.1 and the Cauchy-Schwarz inequality:

$$
\left|\left\langle\hat{\boldsymbol{u}}, \boldsymbol{\delta}_{a}\right\rangle_{B_{a}}^{\Delta \mathcal{C}}\right| \leq\|\Delta \mathcal{C}: \nabla \hat{\boldsymbol{u}}\|_{\boldsymbol{L}^{2}\left(B_{a}\right)}\left\|\boldsymbol{\nabla} \boldsymbol{\delta}_{a}\right\|_{\boldsymbol{L}^{2}\left(B_{a}\right)} \leq C a^{3 / 2} a^{11 / 2} \leq C a^{7} .
$$


Then, the second term of (2.9) admits, as a consequence of the outer expansion (3.20), the expansion

$$
J^{\prime \prime}\left(\boldsymbol{u} ; \boldsymbol{v}_{a}\right)=a^{6} J^{\prime \prime}\left(\boldsymbol{u} ; \boldsymbol{v}_{\text {out }}\right)+o\left(a^{6}\right)
$$

Finally, the remainder $R\left(\boldsymbol{u} ; \boldsymbol{v}_{a}\right)$ in (2.9) can be put in the form (considering only the surface integral component of $J$ for brevity)

$$
R\left(\boldsymbol{u} ; \boldsymbol{v}_{a}\right)=\int_{\Gamma^{\mathrm{m}}}\left\{\int_{0}^{1}(1-t)\left[\nabla_{22} \Psi_{\Gamma}\left(\boldsymbol{x} ; \boldsymbol{u}(\boldsymbol{x})+t \boldsymbol{v}_{a}(\boldsymbol{x})\right)-\nabla_{22} \Psi_{\Gamma}(\boldsymbol{x} ; \boldsymbol{u}(\boldsymbol{x}))\right] \mathrm{d} t\right\}:\left(\boldsymbol{v}_{a} \otimes \boldsymbol{v}_{a}\right)(\boldsymbol{x} ; \boldsymbol{z}) \mathrm{d} S_{x}
$$

The $C^{0, \gamma}$ assumption on $\nabla_{22} \Psi_{\Gamma}$ then yields

$$
\left|R\left(\boldsymbol{u} ; \boldsymbol{v}_{a}\right)\right| \leq C\left\|\boldsymbol{v}_{a}\right\|_{\boldsymbol{L}^{2+\gamma}(\partial \Omega)}^{2+\gamma} \leq C\left\|\boldsymbol{v}_{a}\right\|_{\boldsymbol{H}^{1}(\Omega)}^{2+\gamma} \leq C a^{6+3 \gamma}=o\left(a^{6}\right)
$$

for $a$ small enough, where we have used the fact that the trace mapping defines a continuous $\boldsymbol{H}^{1}(\Omega) \rightarrow \boldsymbol{L}^{p}(\partial \Omega)$ operator for $1 \leq p \leq 4$ (e.g. [15], Thm. 6.6-5), and then invoked Lemma 3.2. The theorem finally follows by using $(4.2 \mathrm{a}-\mathrm{e})$ in $(2.9)$ and grouping all contributions of like orders. Similar arguments, using the pointwise expansion (3.20), apply for components of $J$ defined as integrals over subsets of $\Omega$, if any.

Remark 4.2. Theorem 4.1 uses $\boldsymbol{E}_{p} \bullet \mathcal{A}_{p q} \bullet \boldsymbol{E}_{q}$ with $\boldsymbol{E}_{p}=\boldsymbol{g}_{z}^{p}, \boldsymbol{E}_{q}=\hat{\boldsymbol{g}}_{z}^{q}$, for which $\operatorname{div}\left(\mathcal{C}: \varepsilon\left[\boldsymbol{\varphi}_{p}\left(\boldsymbol{E}_{p}\right)\right]\right)=\mathbf{0}, \operatorname{div}(\mathcal{C}:$ $\left.\varepsilon\left[\boldsymbol{\varphi}_{q}\left(\boldsymbol{E}_{q}\right)\right]\right)=\mathbf{0}$. It can thus also be formulated (for isotropic materials) using the EMTs of [4] (see Rem. 2.4).

\subsection{Centrally symmetric inhomogeneity}

Centrally symmetric inhomogeneity shapes constitute an important category, encompassing many simple shapes such as balls, ellipsoids or cuboids while producing significantly simpler asymptotic expansions as a consequence of the following Lemma:

Lemma 4.3. Let $\mathcal{B}$ be centrally symmetric, i.e. $\boldsymbol{x} \in \mathcal{B} \Longrightarrow-\boldsymbol{x} \in \mathcal{B}$. Let the symmetry operator $\boldsymbol{S}$ be defined by $\boldsymbol{S} \boldsymbol{w}(\boldsymbol{x})=-\boldsymbol{w}(-\boldsymbol{x})$ for any vector function $\boldsymbol{w}: \mathcal{B} \rightarrow \mathbb{R}^{3}$. If equation $\left(\boldsymbol{I}+\mathcal{L}_{\mathcal{B}}\right)\left[\boldsymbol{u}_{\mathcal{B}}\right]=\boldsymbol{u}$ holds (i.e. $\boldsymbol{u}_{\mathcal{B}}$ solves the $\operatorname{FSTP}(2.20)$ with data $\boldsymbol{u})$, then $\left(\boldsymbol{I}+\mathcal{L}_{\mathcal{B}}\right)\left[\boldsymbol{S} \boldsymbol{u}_{\mathcal{B}}\right]=\boldsymbol{S u}$ also holds.

Proof. Let $\overline{\boldsymbol{x}} \in \mathcal{B}$. We write the integral equation $\left(\boldsymbol{I}+\mathcal{L}_{\mathcal{B}}\right)\left[\boldsymbol{u}_{\mathcal{B}}\right]=\boldsymbol{u}$ at the collocation point $-\boldsymbol{x}$ (since by symmetry assumption $-\boldsymbol{x} \in \mathcal{B}$ ) and set $\boldsymbol{\xi}=-\boldsymbol{y}$ for the integration variable of the integral operator $\mathcal{L}_{\mathcal{B}}$, to obtain

$$
\boldsymbol{S} \boldsymbol{u}_{\mathcal{B}}(\boldsymbol{x})-\int_{\mathcal{B}} \nabla \boldsymbol{G}_{\infty}(-\boldsymbol{y}+\boldsymbol{x}): \Delta \mathcal{C}: \nabla \boldsymbol{u}_{\mathcal{B}}(-\boldsymbol{y}) \mathrm{d} V_{y}=\boldsymbol{S} \boldsymbol{u}(\boldsymbol{x}) .
$$

Then, upon noting that (i) $\boldsymbol{\nabla} \boldsymbol{G}_{\infty}(-\boldsymbol{y}+\boldsymbol{x})=-\boldsymbol{\nabla} \boldsymbol{G}_{\infty}(\boldsymbol{y}-\boldsymbol{x})$ by (2.16) and (ii) the definition of $\boldsymbol{S}$ implies that $\boldsymbol{\nabla}\left(\boldsymbol{S} \boldsymbol{u}_{\mathcal{B}}\right)(\boldsymbol{y})=\boldsymbol{\nabla}\left(\boldsymbol{u}_{\mathcal{B}}\right)(-\boldsymbol{y})$, the above equality yields the desired equation $\left(\boldsymbol{I}+\mathcal{L}_{\mathcal{B}}\right)\left[\boldsymbol{S} \boldsymbol{u}_{\mathcal{B}}\right]=\boldsymbol{S} \boldsymbol{u}$ satisfied by $S u_{\mathcal{B}}$.

If $\mathcal{B}$ is centrally symmetric, for any integer $m$, we have $\boldsymbol{S} \boldsymbol{\varphi}_{m}\left[\boldsymbol{E}_{m}\right]=(-1)^{m} \boldsymbol{\varphi}_{m}\left[\boldsymbol{E}_{m}\right]$ (because $\boldsymbol{\varphi}_{m}\left[\boldsymbol{E}_{m}\right]$ is a homogeneous polynomial of degree $m$ ) and $\boldsymbol{S} \boldsymbol{U}_{\mathcal{B}}^{(m)}\left[\boldsymbol{E}_{m}\right]=(-1)^{m} \boldsymbol{U}_{\mathcal{B}}^{(m)}\left[\boldsymbol{E}_{m}\right]$ (by Lemma 4.3). On the other hand, $\langle\boldsymbol{S} \boldsymbol{u}, \boldsymbol{S} \boldsymbol{v}\rangle_{\mathcal{B}}^{\Delta \mathcal{C}}=\langle\boldsymbol{u}, \boldsymbol{v}\rangle_{\mathcal{B}}^{\Delta \mathcal{C}}$ holds for any $\boldsymbol{u}, \boldsymbol{v} \in \boldsymbol{H}^{1}(\mathcal{B})$. Consequently, recalling definition (2.27), the EMTs verify $\mathcal{A}_{p q}=(-1)^{p+q} \mathcal{A}_{p q}$, implying

$$
\mathcal{A}_{p q}=\mathbf{0} \quad \text { whenever } p+q \text { is odd, }
$$

and expressions (4.1a) reduce to

$$
\begin{aligned}
& \mathcal{T}_{3}(\boldsymbol{z})=-\boldsymbol{g}_{z}: \mathcal{A}_{11}: \hat{\boldsymbol{g}}_{z} \\
& \mathcal{T}_{4}(\boldsymbol{z})=0 \\
& \mathcal{T}_{5}(\boldsymbol{z})=-\frac{1}{2} \boldsymbol{g}_{z}: \mathcal{A}_{13} \bullet \hat{\boldsymbol{g}}_{z}^{(3)}-\boldsymbol{g}_{z}^{(2)} \bullet \mathcal{A}_{22} \bullet \hat{\boldsymbol{g}}_{z}^{(2)}-\frac{1}{2} \hat{\boldsymbol{g}}_{z}: \mathcal{A}_{13} \bullet \boldsymbol{g}_{z}^{(3)} \\
& \mathcal{T}_{6}(\boldsymbol{z})=\boldsymbol{g}_{z}: \mathcal{A}_{11}: \nabla_{12} \boldsymbol{G}_{\mathrm{c}}(\boldsymbol{z}, \boldsymbol{z}): \mathcal{A}_{11}: \hat{\boldsymbol{g}}_{z}+\frac{1}{2} J^{\prime \prime}\left(\boldsymbol{u} ; \boldsymbol{v}_{\mathrm{out}}\right)
\end{aligned}
$$


Remark 4.4. Property (4.3) implies that $\boldsymbol{f}_{z}^{(4)}=\mathbf{0}$ in expression (3.16) of $\boldsymbol{V}_{4}$ if $\mathcal{B}$ is centrally-symmetric.

\subsection{Ellipsoidal or spherical inhomogeneity}

Ellipsoids are centrally-symmetrical shapes for which the $\boldsymbol{\varepsilon}\left[\boldsymbol{u}_{p}\left(\boldsymbol{E}_{p}\right)\right]$ are polynomials, see (2.25) for the cases $p=1,2$ of relevance here. As a result, the following closed-form expressions for the elastic moment tensors $\mathcal{A}_{p q}$ can be derived from their definition (2.27):

$$
\mathcal{A}_{11}=|\mathcal{B}| \mathcal{C}:\left(\mathcal{C}+\Delta \mathcal{C}: \mathcal{S}^{1}\right)^{-1}: \Delta \mathcal{C}, \quad \mathcal{A}_{13}=|\mathcal{B}|^{-1} \mathcal{A}_{11} \otimes M, \quad \mathcal{A}_{22}=\widetilde{\mathcal{C}}_{M} \cdot\left(\widetilde{\mathcal{C}}+\widetilde{\Delta \mathcal{C}} \cdot \mathcal{S}^{2}\right)^{-1} \cdot \widetilde{\Delta \mathcal{C}}
$$

where $\boldsymbol{M}$, the geometrical inertia tensor of $\mathcal{B}$, is given by

$$
\boldsymbol{M}=\int_{\mathcal{B}} \overline{\boldsymbol{\xi}} \otimes \overline{\boldsymbol{\xi}} \mathrm{d} \bar{V}
$$

$\widetilde{\Delta \mathcal{C}}$ and $\widetilde{\Delta \mathcal{C}}$ are defined as in (B.2) and $\widetilde{\mathcal{C}}_{M}$ is defined by $\left(\widetilde{\mathcal{C}}_{M}\right)_{i j k \ell m n}=\mathcal{C}_{i j \ell m} M_{k n}$. Formulas (4.5) are then used in expressions $(4.4 \mathrm{a}, \mathrm{d})$ for the topological derivatives.

If $\mathcal{B}$ is in fact the unit sphere, $\boldsymbol{M}=(4 \pi / 15) \boldsymbol{I}$, so that $\mathcal{T}_{5}(\boldsymbol{z})$ is given by

$$
\mathcal{T}_{5}(\boldsymbol{z})=-\frac{1}{10} \Delta \boldsymbol{\nabla} \boldsymbol{u}(\boldsymbol{z}): \mathcal{A}_{11}: \hat{\boldsymbol{g}}_{z}-\boldsymbol{g}_{z}^{(2)} \cdot \mathcal{A}_{22} \bullet \hat{\boldsymbol{g}}_{z}^{(2)}-\frac{1}{10} \Delta \boldsymbol{\nabla} \hat{\boldsymbol{u}}(\boldsymbol{z}): \mathcal{A}_{11}: \boldsymbol{g}_{z}
$$

If, in addition, the background medium is isotropic, the closed-form expressions (2.26a,b) may be used in (4.5) for the Eshelby tensors $\mathcal{S}^{1}, \mathcal{S}^{2}$.

\section{DisCussion}

\subsection{Computational considerations}

The practical evaluation of the topological derivatives $\mathcal{T}_{3}$ to $\mathcal{T}_{6}$ relies on the following ingredients:

(a) Background and adjoint solutions: each needs to be computed just once, irrespective of the number of inhomogeneity sites $\boldsymbol{z}$ considered. As $\mathcal{T}_{5}$ involves derivatives of $\boldsymbol{u}$ and $\hat{\boldsymbol{u}}$ of order up to three (and $\mathcal{T}_{6}$ derivatives of order up to four if $\mathcal{B}$ is not centrally symmetric), suitable solution or post-processing methods are needed. One possibility is to use integral representation formulas for $\boldsymbol{u}(\boldsymbol{z}), \hat{\boldsymbol{u}}(\boldsymbol{z})$, since they can be differentiated at any order.

(b) Polarization tensors: each needs to be computed just once for given inhomogeneity shape and material properties. Their computation requires knowing the FSTP solutions $\boldsymbol{U}_{\mathcal{B}}^{(1)}, \boldsymbol{U}_{\mathcal{B}}^{(2)}$. The latter are known explicitly for ellipsoidal inhomogeneities, except for the need to (i) evaluate Eshelby tensors given by integrals for anisotropic background properties, and (ii) solve numerically a small matrix system for computing $\boldsymbol{U}_{\mathcal{B}}^{(2)}$ via (2.25). For non-ellipsoidal inhomogeneities, one needs to solve numerically the FSTPs $(2.24)$ for $p=1,2$.

(c) Derivatives of either the Green's tensor $\boldsymbol{G}(\cdot, \boldsymbol{z})$ or its complementary part $\boldsymbol{G}_{\mathrm{c}}(\cdot, \boldsymbol{z})$ are needed for the evaluation of $\mathcal{T}_{6}$.

The three fields $\boldsymbol{u}, \hat{\boldsymbol{u}}, \boldsymbol{G}(\cdot, \boldsymbol{z})$ involved in $\mathcal{T}_{3}$ to $\mathcal{T}_{6}$ are defined on the same (background) configuration.

\subsubsection{Evaluations of contributions related to the Green's tensor}

Item (c) above deserves elaboration. If an infinite medium is considered $\left(\Omega=\mathbb{R}^{3}\right.$ ), then of course $\boldsymbol{G}=\boldsymbol{G}_{\infty}$ and $\boldsymbol{G}_{\mathrm{c}}=\mathbf{0}$. In a few other cases, in particular that of a semi-infinite elastic medium with a traction-free plane surface, the Green's tensor $\boldsymbol{G}$ is known in closed form. In most situations, however, contributions of $\boldsymbol{G}(\cdot, \boldsymbol{z})$ or $\boldsymbol{G}_{\mathrm{C}}(\cdot, \boldsymbol{z})$ to the topological derivative $\mathcal{T}_{6}$ will have to be computed numerically. We now briefly discuss the implications of this requirement.

First, the derivatives $\boldsymbol{\nabla}_{1} \boldsymbol{G}(\boldsymbol{z}, \cdot)$ are involved, through (3.20), in $J^{\prime \prime}\left(\boldsymbol{u} ; \boldsymbol{v}_{\text {out }}\right)$; this looks inconvenient as all Green's tensors with source points on $\Gamma^{\mathrm{m}}$ and $\Omega^{\mathrm{m}}$ are a priori needed. However, we note that the well-known 
symmetry property $\boldsymbol{G}(\boldsymbol{z}, \boldsymbol{x})=\boldsymbol{G}^{\mathrm{T}}(\boldsymbol{x}, \boldsymbol{z})$ implies the property $\boldsymbol{\nabla}_{1} \boldsymbol{G}(\boldsymbol{z}, \boldsymbol{x})=\boldsymbol{\nabla}_{2} \boldsymbol{G}^{\mathrm{T}}(\boldsymbol{x}, \boldsymbol{z})$. This allows to evaluate $J^{\prime \prime}\left(\boldsymbol{u} ; \boldsymbol{v}_{\text {out }}\right)$ for given $\boldsymbol{z}$ by means of the Green's tensor having $\boldsymbol{z}$ as source point. Using the additive decomposition (2.14) in (2.13), the complementary Green's tensor $\boldsymbol{G}_{\mathrm{c}}(\cdot, \boldsymbol{z})=\boldsymbol{e}_{k} \otimes \boldsymbol{G}_{\mathrm{c}}^{k}$ is found by solving problems

$$
\operatorname{div}\left(\mathcal{C}: \nabla_{1} G_{\mathrm{c}}^{k}(\cdot, \boldsymbol{z})\right)=\mathbf{0} \quad \text { in } \Omega, \quad \boldsymbol{G}_{\mathrm{c}}^{k}(\cdot, \boldsymbol{z})=-\boldsymbol{G}_{\infty}^{k}(\cdot-\boldsymbol{z}) \quad \text { on } \Gamma_{\mathrm{D}}, \quad \boldsymbol{t}\left[\boldsymbol{G}_{\mathrm{c}}^{k}(\cdot, \boldsymbol{z})\right]=-\boldsymbol{t}\left[\boldsymbol{G}_{\infty}^{k}(\cdot-\boldsymbol{z})\right] \quad \text { on } \Gamma_{\mathrm{N}} .
$$

In fact, the derivatives $\boldsymbol{H}^{k \ell}(\cdot, \boldsymbol{z}):=\partial_{z_{\ell}} \boldsymbol{G}_{\mathrm{c}}(\cdot, \boldsymbol{z})$ can be found as solutions to problems obtained by differentiating the above problem w.r.t. the coordinates of $\boldsymbol{z}$ (a legitimate operation since $\boldsymbol{z}$ acts therein as a parameter):

$$
\operatorname{div}\left(\mathcal{C}: \nabla_{1} \boldsymbol{H}^{k \ell}(\cdot, \boldsymbol{z})\right)=\mathbf{0} \quad \text { in } \Omega, \quad\left\{\begin{array}{cl}
\boldsymbol{H}^{k \ell}(\cdot, \boldsymbol{z})=\partial_{\ell} \boldsymbol{G}_{\infty}^{k}(\cdot-\boldsymbol{z}) & \text { on } \Gamma_{\mathrm{D}}, \\
\boldsymbol{t}\left[\boldsymbol{H}^{k \ell}(\cdot, \boldsymbol{z})\right]=\boldsymbol{t}\left[\partial_{\ell} \boldsymbol{G}_{\infty}^{k}(\cdot-\boldsymbol{z})\right] & \text { on } \Gamma_{\mathrm{N}} .
\end{array}\right.
$$

Problems (5.1) are ordinary elastostatic problems with smooth boundary data (since $\boldsymbol{z} \in \Omega$ ). Then, $\mathcal{T}_{6}(\boldsymbol{z})$ also involves the second-order derivative $\nabla_{12} \boldsymbol{G}_{\mathrm{C}}(\boldsymbol{z}, \boldsymbol{z})$, which can be evaluated using first-order derivatives of $\boldsymbol{\xi} \mapsto \boldsymbol{H}^{k \ell}(\boldsymbol{\xi}, \boldsymbol{z})$ at $\boldsymbol{\xi}=\boldsymbol{z}$.

If the expansion of $\mathbb{J}\left(\mathcal{C}_{a}\right)$ is to be evaluated for many inhomogeneity sites $\boldsymbol{z}$, the induced need to solve many problems of the form (5.1) becomes impractical. One possible remedy then consists in using a (truncated) separable representation of $\boldsymbol{G}_{\infty}(\boldsymbol{\xi}-\boldsymbol{z})$, of the form

$$
\boldsymbol{G}_{\infty}(\boldsymbol{\xi}-\boldsymbol{z})=\sum_{q=1}^{p} \boldsymbol{\alpha}_{q}(\boldsymbol{\xi}) \otimes \boldsymbol{\beta}_{q}(\boldsymbol{z})+\varepsilon_{p}
$$

(such representations are provided by e.g. multipole expansions [20] or the sparse cardinal sine decomposition of [2], with bounds on the truncation error $\varepsilon_{p}$ available). Then, the computational effort associated with solving problems of type (5.1) becomes $O(p)$ irrespective of the number of inhomogeneity sites $\boldsymbol{z}$.

\subsection{Examples of cost functionals}

In addition to the least-squares data misfit functional (2.12), important examples also include the potential energy and reciprocity- or energy-based functionals.

\subsubsection{Potential energy}

The potential energy $\mathbb{E}_{\text {pot }}\left(\mathcal{C}_{a}\right)$ of the solid containing a small inhomogeneity is

$$
\mathbb{E}_{\mathrm{pot}}\left(\mathcal{C}_{a}\right)=\frac{1}{2} \int_{\Gamma_{\mathrm{D}}} \boldsymbol{t}\left[\boldsymbol{u}_{a}\right] \cdot \boldsymbol{u}^{\mathrm{D}} \mathrm{d} S-\frac{1}{2} \int_{\Gamma_{\mathrm{N}}} \boldsymbol{t}^{\mathrm{D}} \cdot \boldsymbol{u}_{a} \mathrm{~d} S
$$

By combining weak-form identities satisfied by $\boldsymbol{u}_{a}$ and $\boldsymbol{u}$ (or, equivalently, by observing that the adjoint solution for $\mathbb{J}=\mathbb{E}_{\text {pot }}$ is $\left.\hat{\boldsymbol{u}}=\frac{1}{2} \boldsymbol{u}\right)$, the potential energy perturbation is found to be given by

$$
\mathbb{E}_{\text {pot }}\left(\mathcal{C}_{a}\right)-\mathbb{E}_{\text {pot }}(\mathcal{C})=\frac{1}{2}\left\langle\boldsymbol{u}, \boldsymbol{u}_{a}\right\rangle_{B_{a}}^{\Delta \mathcal{C}}
$$

The $O\left(a^{6}\right)$ expansion of $\mathbb{E}_{\text {pot }}\left(\mathcal{C}_{a}\right)-\mathbb{E}_{\text {pot }}(\mathcal{C})$ is then easily found from using (4.2b) with $\hat{\boldsymbol{u}}=\boldsymbol{u}$.

\subsubsection{Reciprocity gap}

Some flaw identification methods, based on the concept of reciprocity gap functional (RGF) [7], rely on the availability of the complete Cauchy data on the boundary. For instance, considering the identification of an inhomogeneity defined by $\boldsymbol{z}, B_{a}$ and $\mathcal{C}^{\star}$ from complete knowledge of both $\boldsymbol{u}_{a}$ and $\boldsymbol{t}\left[\boldsymbol{u}_{a}\right]$ on $\partial \Omega$, the relevant RGF is a linear functional $\mathcal{R}: \mathcal{X} \rightarrow \mathbb{R}$ defined by

$$
\mathcal{R}[\boldsymbol{w}]=\int_{\partial \Omega}\left(\boldsymbol{t}\left[\boldsymbol{u}_{a}\right] \cdot \boldsymbol{w}-\boldsymbol{u}_{a} \cdot \boldsymbol{t}[\boldsymbol{w}]\right) \mathrm{d} S
$$


with $\mathcal{X}:=\left\{\boldsymbol{w} \in \boldsymbol{H}^{1}(\Omega), \operatorname{div}(\mathcal{C}: \varepsilon[\boldsymbol{w}])=\mathbf{0}\right\}$. Besides, combining the weak formulations obeyed by $\boldsymbol{u}_{a}$ (with $\boldsymbol{w}$ as test function) and $\boldsymbol{w}$ (with $\boldsymbol{u}_{a}$ as test function), one easily finds

$$
\mathcal{R}[\boldsymbol{w}]=\left\langle\boldsymbol{w}, \boldsymbol{u}_{a}\right\rangle_{B_{a}}^{\Delta \mathcal{C}},
$$

whereupon (4.2b) can again be used to obtain the $O\left(a^{6}\right)$ expansion.

\subsubsection{Energy error}

Energy-based functionals [25] can also be used for e.g. material or flaw identification from overdetermined boundary data. Let $\Gamma_{\mathrm{N}}=S_{\mathrm{o}} \cup S_{\mathrm{no}}$, assuming that a measurement $\boldsymbol{u}_{\text {obs }}$ of the displacement induced in the solid by the excitation $\left(\boldsymbol{u}^{\mathrm{D}}, \boldsymbol{t}^{\mathrm{D}}\right)$ is available on $S_{\mathrm{o}}$. For a solid containing a trial flaw $B_{a}$, one can then define "Neumann" and "Dirichlet" displacement fields $\boldsymbol{u}_{a}^{\mathrm{N}}$ and $\boldsymbol{u}_{a}^{\mathrm{D}}$ that differ only by their boundary data on $S_{\mathrm{o}}$, by

$$
\begin{array}{lll}
\text { Find } \boldsymbol{u}_{a}^{\mathrm{N}} \in \mathcal{V}_{0}, & \left\langle\boldsymbol{u}_{a}^{\mathrm{N}}, \boldsymbol{w}\right\rangle_{\Omega}^{\mathcal{C}_{a}}=F(\boldsymbol{w}) & \forall \boldsymbol{w} \in \mathcal{V}_{0} \\
\text { Find } \boldsymbol{u}_{a}^{\mathrm{D}} \in \mathcal{V}_{0} \text { s.t. } \boldsymbol{u}_{a}=\boldsymbol{u}_{\mathrm{obs}} \text { on } S_{\mathrm{o}}, & \left\langle\boldsymbol{u}_{a}^{\mathrm{D}}, \boldsymbol{w}\right\rangle_{\Omega}^{\mathcal{C}_{a}}=F(\boldsymbol{w}) & \forall \boldsymbol{w} \in \mathcal{V}_{0} \text { s.t. } \boldsymbol{w}=\mathbf{0} \text { on } S_{\mathrm{o}}
\end{array}
$$

A discrepancy between $\boldsymbol{u}_{a}^{\mathrm{N}}$ and $\boldsymbol{u}_{a}^{\mathrm{D}}$ then reveals that the flaw is not correctly identified, and can be defined in terms of the energy error functional

$$
\mathbb{E}\left(\mathcal{C}_{a}\right)=\left\langle\boldsymbol{u}_{a}^{\mathrm{N}}-\boldsymbol{u}_{a}^{\mathrm{D}}, \boldsymbol{u}_{a}^{\mathrm{N}}-\boldsymbol{u}_{a}^{\mathrm{D}}\right\rangle_{\Omega}^{\mathcal{C}_{a}}
$$

It was shown in [13] that

$$
\mathbb{E}\left(\mathcal{C}_{a}\right)-\mathbb{E}(\mathcal{C})=\frac{1}{2}\left\langle\boldsymbol{u}^{\mathrm{D}}, \boldsymbol{u}_{a}^{\mathrm{D}}\right\rangle_{B_{a}}^{\Delta \mathcal{C}}-\frac{1}{2}\left\langle\boldsymbol{u}^{\mathrm{N}}, \boldsymbol{u}_{a}^{\mathrm{N}}\right\rangle_{B_{a}}^{\Delta \mathcal{C}}
$$

with the background fields $\boldsymbol{u}^{\mathrm{N}}, \boldsymbol{u}^{\mathrm{D}}$ defined as above with $\mathcal{C}_{a}=\mathcal{C}$. Then, the $O\left(a^{6}\right)$ approximation of $\mathbb{E}\left(\mathcal{C}_{a}\right)-\mathbb{E}(\mathcal{C})$ is easily found by using (4.2b) with $\left(\hat{\boldsymbol{u}}, \boldsymbol{u}_{a}\right)=\left(\boldsymbol{u}^{\mathrm{D}}, \boldsymbol{u}_{a}^{\mathrm{D}}\right)$ and $\left(\hat{\boldsymbol{u}}, \boldsymbol{u}_{a}\right)=\left(\boldsymbol{u}^{\mathrm{N}}, \boldsymbol{u}_{a}^{\mathrm{N}}\right)$.

\subsection{Multiple inhomogeneities}

All developments presented so far are valid only for the case of a single $a$-dependent inhomogeneity $B_{a}$. We now present, in an abbreviated way, how Theorem 4.1 can be modified to accommodate multiple vanishing inhomogeneities with fixed locations, focusing on the case of two such inhomogeneities (the generalization to three or more being then straightforward). Accordingly, let $B_{a}^{\prime}:=\boldsymbol{z}^{\prime}+a \mathcal{B}^{\prime}$ define the support of a second inhomogeneity, whose material is characterized by the elasticity tensor $\mathcal{C}^{\star \prime} ; B_{a}$ and $B_{a}^{\prime}$ are then scaled by the same length parameter $a$.

For collocation points $\boldsymbol{x} \in B_{a}$, the VIE (2.18) becones

$$
\left(\boldsymbol{I}+\mathcal{L}_{a}\right)\left[\boldsymbol{v}_{a}\right](\boldsymbol{x})+\mathcal{L}_{a}^{\prime}\left[\boldsymbol{v}_{a}^{\prime}\right](\boldsymbol{x})=-\mathcal{L}_{a}[\boldsymbol{u}](\boldsymbol{x})-\mathcal{L}_{a}^{\prime}[\boldsymbol{u}](\boldsymbol{x}), \quad \boldsymbol{x} \in B_{a},
$$

where $\boldsymbol{v}_{a}^{\prime}$ is the restriction of $\boldsymbol{u}_{a}-\boldsymbol{u}$ to $B_{a}^{\prime}$ and

$$
\mathcal{L}_{a}^{\prime}[\boldsymbol{v}]:=\int_{B_{a}^{\prime}} \nabla \boldsymbol{G}_{1}(\boldsymbol{\xi}, \boldsymbol{x}): \Delta \mathcal{C}^{\prime}: \nabla \boldsymbol{v}(\boldsymbol{\xi}) \mathrm{d} V_{\xi},
$$

The VIE formulation for the double-inhomogeneity case then consists of equation (5.2) together with its counterpart obtained by reversing the roles of $B_{a}$ and $B_{a}^{\prime}$.

Using an ansatz of the form (3.6) for each inhomogeneity, the coupling term $\mathcal{L}_{a}^{\prime}\left[\boldsymbol{v}_{a}^{\prime}\right](\boldsymbol{x})$ has a $O\left(a^{3}\right)$ leading contribution, resulting from an outer expansion essentially identical to that of Section 3.2. The lowest-order coefficients $\boldsymbol{V}_{1}, \boldsymbol{V}_{2}$ are therefore still defined by equations (3.13a,b) (with similar equations holding for $\boldsymbol{V}_{1}^{\prime}, \boldsymbol{V}_{2}^{\prime}$ on $\mathcal{B}^{\prime}$ ), and are not influenced by the second inhomogeneity. The expansion of $\mathcal{L}_{a}^{\prime}\left[\boldsymbol{u}+\boldsymbol{v}_{a}^{\prime}\right](\boldsymbol{x})$ is in fact formally 
identical to that of $\mathcal{L}_{a}^{\mathrm{c}}\left[\boldsymbol{u}+\boldsymbol{v}_{a}\right](\boldsymbol{x})$, replacing $\boldsymbol{G}_{\mathrm{c}}(\boldsymbol{\xi}, \boldsymbol{x})$ with $\boldsymbol{G}(\boldsymbol{\xi}, \boldsymbol{x})$ and with all other quantities therein now referring to $B_{a}^{\prime}$, so that we have

$$
\mathcal{P}_{a} \mathcal{L}_{a}^{\prime}\left[\boldsymbol{u}+\boldsymbol{v}_{a}\right](\overline{\boldsymbol{x}})=a^{3} \boldsymbol{f}_{z, z^{\prime}}^{(3)}+a^{4}\left[\boldsymbol{f}_{z, z^{\prime}}^{(4)}+\boldsymbol{L}_{z, z^{\prime}} \cdot \overline{\boldsymbol{x}}\right]+o\left(a^{4}\right)
$$

where $\boldsymbol{f}_{z, z^{\prime}}^{(3)}$ and $\boldsymbol{f}_{z, z^{\prime}}^{(4)}$ are constant vectors, with expressions similar to those of $\boldsymbol{f}_{z}^{(3)}, \boldsymbol{f}_{z}^{(4)}$, that we leave unspecified for brevity, and with

$$
\boldsymbol{L}_{z, z^{\prime}} \cdot \overline{\boldsymbol{x}}=\left(\nabla_{11} \boldsymbol{G}_{\mathrm{C}}\left(\boldsymbol{z}^{\prime}, \boldsymbol{z}\right) \cdot \overline{\boldsymbol{x}}\right): \mathcal{A}_{11}^{\prime}: \boldsymbol{g}_{z^{\prime}} .
$$

The effect of the coupling on $\boldsymbol{V}_{a}$ thus manifests itself through replacing $\boldsymbol{f}_{z}^{(3)}, \boldsymbol{f}_{z}^{(4)}, \boldsymbol{L}_{z}$ with $\boldsymbol{f}_{z}^{(3)}+\boldsymbol{f}_{z, z^{\prime}}^{(3)}, \boldsymbol{f}_{z}^{(4)}+$ $\boldsymbol{f}_{z, z^{\prime}}^{(4)}, \boldsymbol{L}_{z}+\boldsymbol{L}_{z, z^{\prime}}$ in (3.16) (and the symmetric replacements to obtain governing equations for $\boldsymbol{V}_{3}^{\prime}, \boldsymbol{V}_{4}^{\prime}$ ). The expansion of cost functionals is insensitive to the new constants $\boldsymbol{f}_{z, z^{\prime}}^{(3)}$ and $\boldsymbol{f}_{z, z^{\prime}}^{(4)}$ appearing in $\boldsymbol{V}_{a}$ (since inner expansion contributions to $\mathbb{J}\left(\mathcal{C}_{a}\right)$ depend only on $\boldsymbol{\nabla} \boldsymbol{V}_{a}$ and $\left.\boldsymbol{\nabla} \boldsymbol{V}_{a}^{\prime}\right)$. By suitable modifications to the derivation of (4.2b), the topological derivatives $\mathcal{T}_{p}\left(\boldsymbol{z}, \boldsymbol{z}^{\prime}\right)$ for the dual-inhomogeneity configuration are obtained in terms of the topological derivatives $\mathcal{T}_{p}(\boldsymbol{z})$ and $\mathcal{T}_{p}^{\prime}\left(\boldsymbol{z}^{\prime}\right)$ for a single inhomogeneity located at $\boldsymbol{z}$ or $\boldsymbol{z}^{\prime}$, as given by Theorem 4.1, as

$$
\begin{gathered}
\mathcal{T}_{3}\left(\boldsymbol{z}, \boldsymbol{z}^{\prime}\right)=\mathcal{T}_{3}(\boldsymbol{z})+\mathcal{T}_{3}^{\prime}\left(\boldsymbol{z}^{\prime}\right), \quad \mathcal{T}_{4}\left(\boldsymbol{z}, \boldsymbol{z}^{\prime}\right)=\mathcal{T}_{4}(\boldsymbol{z})+\mathcal{T}_{4}^{\prime}\left(\boldsymbol{z}^{\prime}\right), \quad \mathcal{T}_{5}\left(\boldsymbol{z}, \boldsymbol{z}^{\prime}\right)=\mathcal{T}_{5}(\boldsymbol{z})+\mathcal{T}_{5}^{\prime}\left(\boldsymbol{z}^{\prime}\right) \\
\mathcal{T}_{6}\left(\boldsymbol{z}, \boldsymbol{z}^{\prime}\right)=\mathcal{T}_{6}(\boldsymbol{z})+\mathcal{T}_{6}^{\prime}\left(\boldsymbol{z}^{\prime}\right)+2 \boldsymbol{g}_{z}: \mathcal{A}_{11}: \nabla_{11} \boldsymbol{G}\left(\boldsymbol{z}^{\prime}, \boldsymbol{z}\right): \mathcal{A}_{11}^{\prime}: \boldsymbol{g}_{z^{\prime}}+J^{\prime \prime}\left(\boldsymbol{u} ; \boldsymbol{v}_{\text {out }}, \boldsymbol{v}_{\text {out }}^{\prime}\right)
\end{gathered}
$$

In particular, we see that the coupling between inhomogeneities affects only the $O\left(a^{6}\right)$ term (and those beyond that one) in the expansion of $\mathbb{J}\left(\mathcal{C}_{a}\right)$, despite being present in contributions of orders $O\left(a^{3}\right)$ and $O\left(a^{5}\right)$, respectively, in the inner and outer expansions of the displacement field.

\section{Proof of Proposition 2.2}

To establish the bounded invertibility of the integro-differential operators $\boldsymbol{I}+\mathcal{L}_{\mathcal{B}}$, and later that of the integrodifferential operator $\boldsymbol{I}+\mathcal{L}_{a}$ (see Sect. 7), it is useful to reformulate the VIE (2.21) as an integral equation with $\boldsymbol{\sigma}^{\star}:=\Delta \mathcal{C}: \nabla \boldsymbol{u}_{\mathcal{B}}$ as unknown $\left(\boldsymbol{\sigma}^{\star}\right.$ being the equivalent stress associated with the FSTP), as done recently for similar purposes in [22]. Towards this goal, we apply the operator $\Delta \mathcal{C}: \varepsilon=\Delta \mathcal{C}: \nabla$ to the integro-differemtial equation (2.21a). The FSTP then becomes reformulated as

$$
\text { (a) } \mathcal{A}\left[\boldsymbol{\sigma}^{\star}\right](\boldsymbol{x})=\Delta \mathcal{C}_{\mathcal{B}}: \varepsilon[\boldsymbol{u}](\boldsymbol{x}), \quad \text { (b) } \boldsymbol{u}_{\mathcal{B}}(\boldsymbol{x})=\boldsymbol{u}(\boldsymbol{x})-\mathcal{M}\left[\boldsymbol{\sigma}^{\star}\right](\boldsymbol{x}), \quad \boldsymbol{x} \in \mathbb{R}^{3}
$$

where the volume potential $\mathcal{M}$ acting on tensor-valued densities $\boldsymbol{\sigma}^{\star} \in L_{\text {comp }}^{2}\left(\mathbb{R}^{3} ; \mathbb{R}_{\mathrm{sym}}^{3 \times 3}\right)$ is defined by

$$
\mathcal{M}\left[\boldsymbol{\sigma}^{\star}\right](\boldsymbol{x})=\operatorname{div} \int_{\mathbb{R}^{3}} \boldsymbol{G}_{\infty}(\boldsymbol{\xi}-\boldsymbol{x}) \cdot \boldsymbol{\sigma}^{\star}(\boldsymbol{\xi}) \mathrm{d} \bar{V}_{\bar{\xi}}=-\int_{\mathbb{R}^{3}} \boldsymbol{\nabla} \boldsymbol{G}_{\infty}(\boldsymbol{\xi}-\boldsymbol{x}): \boldsymbol{\sigma}^{\star}(\boldsymbol{\xi}) \mathrm{d} \bar{V}_{\bar{\xi}}
$$

and the singular integral operator $\mathcal{A}$ is defined in terms of $\mathcal{M}$ by

$$
\mathcal{A}=\mathcal{I}-\Delta \mathcal{C}_{\mathcal{B}}: \varepsilon[\mathcal{M}]
$$

The potential $\mathcal{M}$ can be shown to verify $\operatorname{div}\left(\mathcal{C}: \varepsilon\left[\mathcal{M}\left(\boldsymbol{\sigma}^{\star}\right)\right]\right)=-\operatorname{div} \boldsymbol{\sigma}^{\star}$, so that the density $\boldsymbol{\sigma}^{\star}$ has the physical meaning of a pre-stress. Since $\nabla \boldsymbol{G}_{\infty} \in L_{\text {loc }}^{1}\left(\mathbb{R}^{3} ; \mathbb{R}^{3 \times 3 \times 3}\right)$, the potential $\mathcal{M}$ is well-defined as a $L_{\text {comp }}^{2}\left(\mathbb{R}^{3} ; \mathbb{R}_{\text {sym }}^{3 \times 3}\right) \rightarrow \boldsymbol{L}_{\text {loc }}^{2}\left(\mathbb{R}^{3}\right)$ operator by virtue of Young's convolution theorem ([14], Thm. 4.15).

The operator $\boldsymbol{\sigma}^{\star} \mapsto \varepsilon\left[\mathcal{M}\left(\boldsymbol{\sigma}^{\star}\right)\right]$ can be given a representation in terms of a singular integral operator involving the kernel $\boldsymbol{H}_{\infty}$, defined in component notation by

$$
\left(H_{\infty}\right)_{i j k \ell}=\frac{1}{4}\left(G_{i k, j \ell}+G_{i \ell, j k}+G_{j k, i \ell}+G_{j \ell, i k}\right),
$$


and whose singularity at the origin is not integrable (see e.g. [22], Sect. 4). Definition (6.3) of $\boldsymbol{H}_{\infty}$ as the symmetrized version of $\boldsymbol{\nabla} \boldsymbol{\nabla} \boldsymbol{G}_{\infty}$ is consistent with the symmetry of $\boldsymbol{\sigma}^{\star}$ (by assumption) and of $\boldsymbol{\varepsilon}\left[\mathcal{M}\left(\boldsymbol{\sigma}^{\star}\right)\right]$. The precise singular integral operator form of $\sigma^{\star} \mapsto \varepsilon\left[\mathcal{M}\left(\boldsymbol{\sigma}^{\star}\right)\right]$ will not be needed here.

From the formulation $(6.1, \mathrm{~b})$ of the FSTP and since $\boldsymbol{u} \mapsto \Delta \mathcal{C}_{\mathcal{B}}: \varepsilon[\boldsymbol{u}]$ is a continuous $\boldsymbol{H}^{1}(\mathcal{B}) \rightarrow \boldsymbol{L}^{2}(\mathcal{B})$ mapping, Proposition 2.2 requires proving that (i) $\mathcal{M}: \boldsymbol{L}^{2}(\mathcal{B}) \rightarrow \boldsymbol{H}^{1}(\mathcal{B})$ is bounded and (ii) $\mathcal{A}: \boldsymbol{L}^{2}(\mathcal{B}) \rightarrow \boldsymbol{L}^{2}(\mathcal{B})$ is invertible with bounded inverse. Step (i) is a known result on elastic volume potentials that can be obtained from the theory of pseudodifferential operators (see e.g. [24], Thm. 6.1.12); we nevertheless give a proof as some of its ingredients will then be used for step (ii).

Step (i): boundedness of $\mathcal{M}: \boldsymbol{L}^{2}(\mathcal{B}) \rightarrow \boldsymbol{H}^{1}(\mathcal{B})$.

We already know that $\mathcal{M}: L_{\text {comp }}^{2}\left(\mathbb{R}^{3} ; \mathbb{R}_{\text {sym }}^{3 \times 3}\right) \rightarrow \boldsymbol{L}_{\text {loc }}^{2}\left(\mathbb{R}^{3}\right)$ is bounded, so only need to show the boundedness of $\varepsilon[\mathcal{M}]: L_{\text {comp }}^{2}\left(\mathbb{R}^{3} ; \mathbb{R}_{\text {sym }}^{3 \times 3}\right) \rightarrow \boldsymbol{L}_{\text {loc }}^{2}\left(\mathbb{R}^{3}\right)$.

The kernel $\nabla \boldsymbol{G}_{\infty}$ defines a tempered distribution (since $\nabla \boldsymbol{G}_{\infty}$ is locally summable and belongs to the class of slowly growing functions), making the convolution $\mathcal{M}\left[\boldsymbol{\sigma}^{\star}\right]=\left(\varepsilon\left[\boldsymbol{G}_{\infty}\right]\right) \star \boldsymbol{\sigma}^{\star}$ well defined for any $\boldsymbol{\sigma}^{\star} \in$ $L_{\text {comp }}^{2}\left(\mathbb{R}^{3} ; \mathbb{R}_{\text {sym }}^{3 \times 3}\right)$. Therefore, $\varepsilon[\mathcal{M}]\left(\boldsymbol{\sigma}^{\star}\right)=\boldsymbol{H}_{\infty} \star \boldsymbol{\sigma}^{\star}$ also holds in the sense of distributions. Moreover, under the present conditions, the distributional version of the Fourier convolution theorem applies:

$$
\mathcal{F}\left[\varepsilon[\mathcal{M}]\left(\boldsymbol{\sigma}^{\star}\right)\right](\boldsymbol{\rho})=\mathcal{F}\left[\boldsymbol{H}_{\infty} \star \boldsymbol{\sigma}^{\star}\right](\boldsymbol{\rho})=\mathcal{F}\left[\boldsymbol{H}_{\infty}\right](\boldsymbol{\rho}): \mathcal{F}\left[\boldsymbol{\sigma}^{\star}\right](\boldsymbol{\rho})
$$

with the Fourier transform $\mathcal{F}$ defined such that $\mathcal{F}[f](\boldsymbol{\rho})=\int_{\mathbb{R}^{3}} e^{-2 \pi \mathrm{i} \boldsymbol{x} \cdot \boldsymbol{\rho}} f(\boldsymbol{x}) \mathrm{d} V_{x}$ whenever $f \in L^{1}\left(\mathbb{R}^{3}\right)$. Moreover, $\hat{\boldsymbol{H}}(\boldsymbol{\rho}):=\mathcal{F}\left[\boldsymbol{H}_{\infty}\right](\boldsymbol{\rho})$ is given for $\boldsymbol{\rho} \neq \mathbf{0}$ by the well-known expression

$$
\hat{H}_{i j k \ell}(\boldsymbol{\rho})=-\frac{1}{4}\left[Q_{j \ell}(\boldsymbol{\rho}) \rho_{i} \rho_{k}+Q_{i \ell}(\boldsymbol{\rho}) \rho_{j} \rho_{k}+Q_{j k}(\boldsymbol{\rho}) \rho_{i} \rho_{\ell}+Q_{i k}(\boldsymbol{\rho}) \rho_{j} \rho_{\ell},\right]
$$

where $\boldsymbol{Q}(\boldsymbol{\rho})=\boldsymbol{N}(\boldsymbol{\rho})^{-1}$ and $\boldsymbol{N}(\boldsymbol{\rho})$ is the (symmetric, positive definite) acoustic tensor defined by $N_{i k}(\boldsymbol{\rho})=$ $C_{i j k \ell} \rho_{j} \rho_{\ell}[31]$. In particular, $\hat{\boldsymbol{H}}(\boldsymbol{\rho})$ is $C^{\infty}\left(\mathbb{R}^{3} \backslash\{\mathbf{0}\}\right)$ and homogeneous with degree 0 in $\rho$, i.e. $\hat{\boldsymbol{H}}(\boldsymbol{\rho})=\hat{\boldsymbol{H}}(\hat{\boldsymbol{\rho}})$ (with $\hat{\boldsymbol{\rho}}:=\boldsymbol{\rho} /|\boldsymbol{\rho}|)$. It is therefore bounded in $\mathbb{R}^{3}$, and the boundedness of $\varepsilon[\mathcal{M}]: L_{\text {comp }}^{2}\left(\mathbb{R}^{3} ; \mathbb{R}_{\mathrm{sym}}^{3 \times 3}\right) \rightarrow \boldsymbol{L}_{\text {loc }}^{2}\left(\mathbb{R}^{3}\right.$ ) follows with the help of Plancherel's theorem:

$$
\left.\left\|\varepsilon[\mathcal{M}]\left(\boldsymbol{\sigma}^{\star}\right)\right\|_{\boldsymbol{L}^{2}\left(\mathbb{R}^{3}\right)}=\left\|\mathcal{F}\left(\varepsilon[\mathcal{M}]\left(\boldsymbol{\sigma}^{\star}\right)\right)\right\|_{L^{2}\left(\mathbb{R}^{3}\right)}=\left\|\hat{\boldsymbol{H}}: \mathcal{F}\left[\boldsymbol{\sigma}^{\star}\right]\right\|_{\boldsymbol{L}^{2}\left(\mathbb{R}^{3}\right)} \leq C \| \mathcal{F}\left[\boldsymbol{\sigma}^{\star}\right]\right]\left\|_{L^{2}\left(\mathbb{R}^{3}\right)}=C\right\| \boldsymbol{\sigma}^{\star} \|_{\boldsymbol{L}^{2}\left(\mathbb{R}^{3}\right)}
$$

Remark 6.1. The definition (6.4) of $\hat{\boldsymbol{H}}$ requires $\mathcal{C}$ to be nonzero (making $\boldsymbol{N}(\boldsymbol{\rho})$ well-defined and invertible by virtue of the positive definiteness of $\mathcal{C}$ ) and bounded (making $\boldsymbol{Q}(\boldsymbol{\rho})$ also bounded for given $\boldsymbol{\rho}$ ).

Step (ii): Bounded invertibility of $\mathcal{A}: \boldsymbol{L}^{2}(\mathcal{B}) \rightarrow \boldsymbol{L}^{2}(\mathcal{B})$.

The (tensor-valued) symbol $\boldsymbol{\Psi}(\boldsymbol{x}, \hat{\boldsymbol{\rho}})$ of the singular integral operator $\mathcal{A}$ defined by (6.2) is given in terms of $\mathcal{F}\left[\boldsymbol{H}_{\infty}\right]$ by $([30]$, Sect. 11.1)

$$
\boldsymbol{\Psi}(\boldsymbol{x}, \hat{\boldsymbol{\rho}})=\mathcal{I}-\Delta \mathcal{C}_{\mathcal{B}}(\boldsymbol{x}): \hat{\boldsymbol{H}}(\hat{\boldsymbol{\rho}}),
$$

and can be given a $6 \times 6$ matrix representation $[\boldsymbol{\Psi}(\boldsymbol{x}, \hat{\boldsymbol{\rho}})]_{6 \times 6}$. Moreover, the identity

$$
\left[\mathcal{I}+\Delta \mathcal{C}_{\mathcal{B}}(\boldsymbol{x}): \hat{\boldsymbol{H}}^{\star}\right]:\left[\mathcal{I}-\Delta \mathcal{C}_{\mathcal{B}}(\boldsymbol{x}): \hat{\boldsymbol{H}}\right]=\mathcal{I}
$$

holds [19], where $\hat{\boldsymbol{H}}^{\star}$ is defined by (6.4) with $\mathcal{C}$ replaced by $\mathcal{C}^{\star}$ and as a consequence of

$$
\hat{\boldsymbol{H}}^{\star}: \Delta \mathcal{C}: \hat{\boldsymbol{H}}=\Delta \mathcal{C}:\left(\hat{\boldsymbol{H}}^{\star}-\hat{\boldsymbol{H}}\right),
$$

which can be checked by straightforward algebra using (6.4). This identity shows that the symbol tensor $\boldsymbol{\Psi}(\boldsymbol{x}, \hat{\boldsymbol{\rho}})$ is invertible with its inverse given by $\boldsymbol{\Psi}^{-1}(\boldsymbol{x}, \hat{\boldsymbol{\rho}})=\mathcal{I}+\Delta \mathcal{C}_{\mathcal{B}}(\boldsymbol{x}): \hat{\boldsymbol{H}}^{\star}(\hat{\boldsymbol{\rho}})$ (a corresponding invertibility result of 
course holding for the symbol matrix $\left.[\boldsymbol{\Psi}(\boldsymbol{x}, \hat{\boldsymbol{\rho}})]_{6 \times 6}\right)$. We can then rely on the following result from Mikhlin's theory for singular integral equations:

Lemma 6.2 ([30], Chap. 14, Theorem 5.2). Let $\mathcal{A}$ be a singular matrix operator and $[\boldsymbol{\Psi}(\boldsymbol{x}, \hat{\boldsymbol{\rho}})]_{n \times n}$ its $n \times n$ symbol matrix. If the moduli of the minors

$$
D_{1}=\Psi_{11}(\boldsymbol{x}, \hat{\boldsymbol{\rho}}), \quad D_{2}=\left|\begin{array}{c}
\Psi_{11}(\boldsymbol{x}, \hat{\boldsymbol{\rho}}) \Psi_{12}(\boldsymbol{x}, \hat{\boldsymbol{\rho}}) \\
\Psi_{21}(\boldsymbol{x}, \hat{\boldsymbol{\rho}}) \Psi_{22}(\boldsymbol{x}, \hat{\boldsymbol{\rho}})
\end{array}\right|, \ldots, \quad D_{n}=\left|\begin{array}{cc}
\Psi_{11}(\boldsymbol{x}, \hat{\boldsymbol{\rho}}) \ldots \Psi_{1 n}(\boldsymbol{x}, \hat{\boldsymbol{\rho}}) \\
\vdots \\
\Psi_{n 1}(\boldsymbol{x}, \hat{\boldsymbol{\rho}}) \ldots \Psi_{n n}(\boldsymbol{x}, \hat{\boldsymbol{\rho}})
\end{array}\right|
$$

are all bounded from below by a positive constant almost everywhere for $(\boldsymbol{x}, \hat{\boldsymbol{\rho}}) \in \mathbb{R}^{3} \times \hat{S}$, the operator $\mathcal{A}$ is Fredholm with index 0 (as defined in e.g. [29], Chap. 2 or [32]).

Since (i) $\hat{\boldsymbol{\rho}} \mapsto[\boldsymbol{\Psi}(\boldsymbol{x}, \hat{\boldsymbol{\rho}})]_{6 \times 6}$ is (together with all its minors) continuous on the (compact) unit sphere and (ii) $\boldsymbol{x} \mapsto[\boldsymbol{\Psi}(\boldsymbol{x}, \hat{\boldsymbol{\rho}})]_{6 \times 6}$ is piecewise-constant, the invertibility of $[\boldsymbol{\Psi}(\boldsymbol{x}, \hat{\boldsymbol{\rho}})]_{6 \times 6}$ for each $(\boldsymbol{x}, \hat{\boldsymbol{\rho}})$ guarantees that all minors involved in Lemma 6.2 are nonzero and bounded away from zero (possibly after applying a suitable column permutation to $\left.[\boldsymbol{\Psi}(\boldsymbol{x}, \hat{\boldsymbol{\rho}})]_{6 \times 6}\right)$. The condition of Lemma (6.2) being fulfilled, the operator $\mathcal{A}$ is bounded and Fredholm with index 0 .

The solution to the FSTP (2.20) is known to be unique, and solves problem (6.1); conversely, the property $\operatorname{div}\left(\mathcal{C}: \varepsilon\left[\mathcal{M}\left(\boldsymbol{\sigma}^{\star}\right)\right]\right)=-\operatorname{div} \boldsymbol{\sigma}^{\star}$ and the decay at infinity of $\mathcal{M}\left[\boldsymbol{\sigma}^{\star}\right]$ imply that any solution of (6.1) solves (2.20). Problem (6.1) therefore has at most one solution. Concluding, as a Fredholm operator with index 0, $\mathcal{A}$ : $L^{2}\left(\mathcal{B} ; \mathbb{R}_{\mathrm{sym}}^{3 \times 3}\right) \rightarrow L^{2}\left(\mathcal{B} ; \mathbb{R}_{\mathrm{sym}}^{3 \times 3}\right)$ is invertible with bounded inverse (by virtue of e.g. [29], Thm. 2.1 and Cor. 2.2).

\section{Proof of Proposition 3.1}

As a consequence of equation (2.18) satisfied by $\boldsymbol{v}_{a}$, the inner expansion error $\boldsymbol{\delta}_{a}:=\boldsymbol{v}_{a}-\mathcal{P}_{a}^{-1}\left[\boldsymbol{V}_{a}\right] \in \boldsymbol{H}^{1}\left(B_{a}\right)$ solves the integral equation

$$
\left(\boldsymbol{I}+\mathcal{L}_{a}\right)\left[\boldsymbol{\delta}_{a}\right]=\boldsymbol{\gamma}_{a}, \quad \text { with } \quad \boldsymbol{\gamma}_{a}=-\mathcal{L}_{a}[\boldsymbol{u}]-\left(\boldsymbol{I}+\mathcal{L}_{a}\right) \mathcal{P}_{a}^{-1}\left[\boldsymbol{V}_{a}\right]
$$

Proposition 3.1 follows directly from the following two Lemmas.

Lemma 7.1. Assume that the background and inhomogeneity elasticity tensors $\mathcal{C}$ and $\mathcal{C}^{\star}$ are both positive definite and bounded. The integro-differential operator $\boldsymbol{I}+\mathcal{L}_{a}: \boldsymbol{H}^{1}\left(B_{a}\right) \rightarrow \boldsymbol{H}^{1}\left(B_{a}\right)$ is invertible. Moreover, there exists $a_{1}>0$ such that $\left(\boldsymbol{I}+\mathcal{L}_{a}\right)^{-1}$ is bounded uniformly in a for all $a \leq a_{1}$.

Lemma 7.2. The right-hand side $\boldsymbol{\gamma}_{a}$ of equation (7.1) satisfies $\left\|\boldsymbol{\gamma}_{a}\right\|_{\boldsymbol{H}^{1}\left(B_{a}\right)}=O\left(a^{11 / 2}\right)$.

\subsection{Proof of Lemma 7.1}

We invoke the decomposition $\mathcal{L}_{a}=\mathcal{L}_{a}^{\infty}+\mathcal{L}_{a}^{\mathrm{c}}$ induced by the decomposition $\boldsymbol{G}=\boldsymbol{G}_{\infty}+\boldsymbol{G}_{\mathrm{c}}$ of the Green's tensor, see (3.8). Recalling that $\mathcal{L}_{a}^{\infty}=\mathcal{P}_{a}^{-1} \mathcal{L}_{\mathcal{B}} \mathcal{P}_{a}$, let $\mathcal{H}_{a}:=\boldsymbol{I}+\mathcal{P}_{a}^{-1} \mathcal{L}_{\mathcal{B}} \mathcal{P}_{a}$ denote the leading term in the expansion of $\boldsymbol{I}+\mathcal{L}_{a}$ in powers of $a$, such that $\boldsymbol{I}+\mathcal{L}_{a}=\mathcal{H}_{a}+\mathcal{L}_{a}^{\mathrm{c}}$.

In a first step, we show bounded invertibility of $\mathcal{H}_{a}: \boldsymbol{H}^{1}\left(B_{a}\right) \rightarrow \boldsymbol{H}^{1}\left(B_{a}\right)$ for sufficiently small $a$, uniformly in $a$. Invertibility of $\mathcal{H}_{a}$ results from Proposition 2.2 and $\mathcal{L}_{a}^{\infty}=\mathcal{P}_{a}^{-1} \mathcal{L}_{\mathcal{B}} \mathcal{P}_{a}$. To estimate $\left\|\left(\mathcal{L}_{a}^{\infty}\right)^{-1}\right\|$, we consider the equation $\mathcal{H}_{a}\left[\boldsymbol{u}_{a}\right]=\boldsymbol{u}$, for some given background displacement $\boldsymbol{u} \in \boldsymbol{H}^{1}\left(B_{a}\right)$. This equation is equivalent to $\left(\boldsymbol{I}+\mathcal{L}_{\mathcal{B}}\right) \mathcal{P}_{a}\left[\boldsymbol{u}_{a}\right]=\mathcal{P}_{a}[\boldsymbol{u}]$, so can be solved using steps (6.1a,b), wherein operators $\mathcal{A}^{-1}$ and $\mathcal{M}$ are known to be bounded (Sect. 6). Step (6.1a) gives $\left\|\boldsymbol{\nabla} \mathcal{P}_{a} \boldsymbol{u}_{a}\right\|_{\boldsymbol{L}^{2}\left(B_{a}\right)} \leq\left\|\boldsymbol{\nabla} \mathcal{P}_{a} \boldsymbol{u}\right\|_{\boldsymbol{L}^{2}\left(B_{a}\right)}$ for some $C>0$. Using (3.4), this implies

$$
\left\|\boldsymbol{\nabla} \boldsymbol{u}_{a}\right\|_{\boldsymbol{L}^{2}\left(B_{a}\right)} \leq\|\boldsymbol{\nabla} \boldsymbol{u}\|_{\boldsymbol{L}^{2}\left(B_{a}\right)}
$$


Then, Step (6.1b) yields $\left\|\mathcal{P}_{a} \boldsymbol{u}_{a}\right\|_{\boldsymbol{L}^{2}(\mathcal{B})} \leq\left\|\mathcal{P}_{a} \boldsymbol{u}\right\|_{\boldsymbol{L}^{2}(\mathcal{B})}+C\left\|\boldsymbol{\nabla}_{\mathcal{P}_{a}} \boldsymbol{u}\right\|_{\boldsymbol{L}^{2}(\mathcal{B})}$ for some $C>0$, which implies (invoking (3.4) again) $\left\|\boldsymbol{u}_{a}\right\|_{\boldsymbol{L}^{2}(\mathcal{B})} \leq\|\boldsymbol{u}\|_{\boldsymbol{L}^{2}(\mathcal{B})}+C\|\boldsymbol{\nabla} \boldsymbol{u}\|_{\boldsymbol{L}^{2}(\mathcal{B})}$. Choosing $a_{0}$ such that $B_{a} \Subset \Omega$ for any $a \leq a_{0}$, we therefore have

$$
\left\|\boldsymbol{u}_{a}\right\|_{\boldsymbol{L}^{2}\left(B_{a}\right)} \leq \max \left(1, a_{0} C\right)\|\boldsymbol{u}\|_{\boldsymbol{H}^{1}\left(B_{a}\right)} \quad \text { for any } a \leq a_{0}
$$

Summing inequalities (7.2a,b), we find that there exists $C_{0}$ such that $\left\|\boldsymbol{\nabla} \boldsymbol{u}_{a}\right\|_{\boldsymbol{H}^{1}\left(B_{a}\right)} \leq C_{0}\|\boldsymbol{\nabla} \boldsymbol{u}\|_{\boldsymbol{H}^{1}\left(B_{a}\right)}$, i.e. that $\mathcal{H}_{a}^{-1}=\mathcal{P}_{a}^{-1}\left(\boldsymbol{I}+\mathcal{L}_{\mathcal{B}}\right)^{-1} \mathcal{P}_{a}: \boldsymbol{H}^{1}\left(B_{a}\right) \rightarrow \boldsymbol{H}^{1}\left(B_{a}\right)$ is bounded uniformly in $a$, for $a \leq a_{0}$.

The second step consists in writing $\boldsymbol{I}+\mathcal{L}_{a}=\mathcal{H}_{a}\left[\boldsymbol{I}+\mathcal{H}_{a}^{-1} \mathcal{L}_{a}^{\mathrm{c}}\right]$ and showing that $\boldsymbol{I}+\mathcal{H}_{a}^{-1} \mathcal{L}_{a}^{\mathrm{c}}$ is invertible using Neumann series for $a$ small enough. Since $\mathcal{L}_{a}^{\mathrm{c}}$ is an integral operator with $C^{\infty}(\Omega \times \Omega)$ kernel $\boldsymbol{\nabla} \boldsymbol{G}_{\mathrm{c}}$, its norm can be readily estimated using the Cauchy-Schwarz inequality:

$$
\begin{aligned}
\left\|\mathcal{L}_{a}^{\mathrm{c}}[\boldsymbol{v}]\right\|_{\boldsymbol{H}^{1}\left(B_{a}\right)}^{2} & \leq\left(\left\|\boldsymbol{\nabla}_{2} \boldsymbol{G}_{\mathrm{C}}\right\|_{\boldsymbol{L}^{2}\left(B_{a} \times B_{a}\right)}^{2}+\left\|\boldsymbol{\nabla}_{12} \boldsymbol{G}_{\mathrm{c}}\right\|_{\boldsymbol{L}^{2}\left(B_{a} \times B_{a}\right)}^{2}\right)\|\Delta \mathcal{C}: \boldsymbol{\varepsilon}[\boldsymbol{v}]\|_{\boldsymbol{L}^{2}\left(B_{a}\right)}^{2} \\
& \leq a^{6} C_{\mathrm{C}}^{2}\|\boldsymbol{\nabla} \boldsymbol{v}\|_{\boldsymbol{L}^{2}\left(B_{a}\right)}^{2} \leq a^{6} C_{\mathrm{C}}^{2}\|\boldsymbol{v}\|_{\boldsymbol{H}^{1}\left(B_{a}\right)}^{2},
\end{aligned}
$$

so that $\left\|\mathcal{L}_{a}^{\mathrm{c}}\right\| \leq a^{3} C_{\mathrm{C}}$. Consequently, for any $c<1$ there exists an inclusion size $a_{1}$ such that $\left\|\mathcal{H}_{a}^{-1} \mathcal{L}_{a}^{\mathrm{c}}\right\| \leq c<1$ for any $a \leq a_{1}$, namely:

$$
a_{1}=\min \left(a_{0}, c^{1 / 3}\left(C_{0} C_{\mathrm{C}}\right)^{-1 / 3}\right)
$$

As a result, for any $a<a_{0}, \boldsymbol{I}+\mathcal{H}_{a}^{-1} \mathcal{L}_{a}^{\mathrm{c}}$ is invertible by Neumann series, with bounded inverse:

$$
\left\|\left(\boldsymbol{I}+\mathcal{H}_{a}^{-1} \mathcal{L}_{a}^{\mathrm{c}}\right)^{-1}\right\| \leq \frac{1}{1-\left\|\mathcal{H}_{a}^{-1} \mathcal{L}_{a}^{\mathrm{c}}\right\|} \leq \frac{1}{1-c}
$$

Concluding, $\boldsymbol{I}+\mathcal{L}_{a}: \boldsymbol{H}^{1}\left(B_{a}\right) \rightarrow \boldsymbol{H}^{1}\left(B_{a}\right)$ is invertible, with its inverse given by $\left(\boldsymbol{I}+\mathcal{L}_{a}\right)^{-1}=\left[\boldsymbol{I}+\mathcal{H}_{a} \mathcal{L}_{a}^{\mathrm{c}}\right]^{-1} \mathcal{H}_{a}^{-1}$ and bounded uniformly in $a$, for all $a<a_{1}$.

\subsection{Proof of Lemma 7.2}

The right-hand side $\gamma_{a}$ defined by (7.1) is evaluated by recalling that $\boldsymbol{V}_{1}, \ldots \boldsymbol{V}_{4}$ are given in (3.14), (3.16) (or, equivalently, satisfy Eqs. $(3.13 \mathrm{a}, \mathrm{b}),(3.15 \mathrm{a}, \mathrm{b})$ ), applying wherever necessary the inverse mapping $\mathcal{P}_{a}^{-1}$, and rearranging contributions, to obtain

$$
\begin{gathered}
\boldsymbol{\gamma}_{a}(\boldsymbol{x})=-\mathcal{M}\left[\Delta \mathcal{C}: \boldsymbol{F}_{0}\right](\boldsymbol{x})-\sum_{m=1}^{4}\left\langle\boldsymbol{F}_{m}, \boldsymbol{H}_{m}(\boldsymbol{x}, \cdot)\right\rangle_{B_{a}}^{\Delta \mathcal{C}} \\
\nabla \boldsymbol{\gamma}_{a}(\boldsymbol{x})=-\boldsymbol{\nabla} \mathcal{M}\left[\Delta \mathcal{C}: \boldsymbol{F}_{0}\right](\boldsymbol{x})-\sum_{m=1}^{4} \boldsymbol{\nabla}\left\langle\boldsymbol{F}_{m}, \boldsymbol{H}_{m}(\boldsymbol{x}, \cdot)\right\rangle_{B_{a}}^{\Delta \mathcal{C}}
\end{gathered}
$$

with

$$
\begin{aligned}
& \boldsymbol{F}_{0}(\boldsymbol{\xi})=\nabla \boldsymbol{u}(\boldsymbol{\xi})-\left(\boldsymbol{g}_{z}+\boldsymbol{g}_{z}^{(2)} \cdot(\boldsymbol{\xi}-\boldsymbol{z})+\frac{1}{2} \boldsymbol{g}_{z}^{(3)}:(\boldsymbol{\xi}-\boldsymbol{z})^{\otimes 2}+\frac{1}{6} \boldsymbol{g}_{z}^{(4)} \bullet(\boldsymbol{\xi}-\boldsymbol{z})^{\otimes 3}\right), \\
& \boldsymbol{F}_{1}(\boldsymbol{\xi})=\nabla \boldsymbol{u}(\boldsymbol{\xi})-\left(\boldsymbol{g}_{z}+\boldsymbol{g}_{z}^{(2)} \cdot(\boldsymbol{\xi}-\boldsymbol{z})\right), \\
& \boldsymbol{F}_{2}(\boldsymbol{\xi})=\frac{a^{2}}{2} \mathcal{P}_{a}^{-1} \boldsymbol{\nabla} \boldsymbol{V}_{\mathcal{B}}^{(3)}\left[\boldsymbol{g}_{z}^{(3)}\right](\boldsymbol{\xi})+a^{3}\left(\frac{1}{6} \mathcal{P}_{a}^{-1} \nabla \boldsymbol{V}_{\mathcal{B}}^{(4)}\left[\boldsymbol{g}_{z}^{(4)}\right](\boldsymbol{\xi})-\mathcal{P}_{a}^{-1} \nabla \boldsymbol{U}_{\mathcal{B}}^{(1)}\left[\boldsymbol{L}_{z}\right](\boldsymbol{\xi})\right), \\
& \boldsymbol{F}_{3}(\boldsymbol{\xi})=\boldsymbol{g}_{z}+\mathcal{P}_{a}^{-1} \nabla \boldsymbol{V}_{\mathcal{B}}^{(1)}\left[\boldsymbol{g}_{z}\right](\boldsymbol{\xi}), \\
& \boldsymbol{F}_{4}(\boldsymbol{\xi})=\boldsymbol{g}_{z}^{(2)} \cdot(\boldsymbol{\xi}-\boldsymbol{z})+a \mathcal{P}_{a}^{-1} \boldsymbol{\nabla} \boldsymbol{V}_{\mathcal{B}}^{(2)}\left[\boldsymbol{g}_{z}^{(2)}\right](\boldsymbol{\xi}),
\end{aligned}
$$

and with the smooth kernels $\boldsymbol{H}_{m}$ defined by

$$
\begin{aligned}
& \boldsymbol{H}_{1}(\boldsymbol{\xi}, \boldsymbol{x})=\boldsymbol{H}_{2}(\boldsymbol{\xi}, \boldsymbol{x})=\boldsymbol{\nabla}_{1} \boldsymbol{G}_{\mathrm{c}}(\boldsymbol{\xi}, \boldsymbol{x}) \\
& \boldsymbol{H}_{3}(\boldsymbol{\xi}, \boldsymbol{x})=\nabla_{1} \boldsymbol{G}_{\mathrm{c}}(\boldsymbol{\xi}, \boldsymbol{x})-\boldsymbol{\nabla}_{1} \boldsymbol{G}_{\mathrm{c}}(\boldsymbol{z}, \boldsymbol{z})-a\left(\boldsymbol{\nabla}_{11} \boldsymbol{G}_{\mathrm{c}}(\boldsymbol{z}, \boldsymbol{z}) \cdot \overline{\boldsymbol{\xi}}+\nabla_{12} \boldsymbol{G}_{\mathrm{C}}(\boldsymbol{z}, \boldsymbol{z}) \cdot \overline{\boldsymbol{x}}\right) \\
& \boldsymbol{H}_{4}(\boldsymbol{\xi}, \boldsymbol{x})=\boldsymbol{\nabla}_{1} \boldsymbol{G}_{\mathrm{c}}(\boldsymbol{\xi}, \boldsymbol{x})-\boldsymbol{\nabla}_{1} \boldsymbol{G}_{\mathrm{c}}(\boldsymbol{z}, \boldsymbol{z}) .
\end{aligned}
$$


We begin by noting that the estimates

$$
\begin{array}{lll}
\left\|\boldsymbol{F}_{0}\right\|_{\boldsymbol{L}^{2}\left(B_{a}\right)}=O\left(a^{11 / 2}\right), & \left\|\boldsymbol{F}_{1}\right\|_{\boldsymbol{L}^{2}\left(B_{a}\right)}=O\left(a^{7 / 2}\right), & \left\|\boldsymbol{F}_{2}\right\|_{\boldsymbol{L}^{2}\left(B_{a}\right)}=O\left(a^{7 / 2}\right), \\
\left\|\boldsymbol{F}_{3}\right\|_{\boldsymbol{L}^{2}\left(B_{a}\right)}=O\left(a^{3 / 2}\right), & \left\|\boldsymbol{F}_{4}\right\|_{\boldsymbol{L}^{2}\left(B_{a}\right)}=O\left(a^{5 / 2}\right) &
\end{array}
$$

hold (the first two by virtue of $\boldsymbol{F}_{0}$ and $\boldsymbol{F}_{1}$ being remainders of Taylor expansions of the background solution $\boldsymbol{u}$, which by assumption has continuous derivatives of sufficient order in $\bar{B}_{a}$, see Rem. 2.1).

The known boundedness of $\mathcal{M}$ (see Sect. 6) then implies, for some constant $C>0$, that

$$
\left\|\mathcal{M}\left[\Delta \mathcal{C}: \boldsymbol{F}_{0}\right]\right\|_{\boldsymbol{H}^{1}\left(B_{a}\right)} \leq C\left\|\boldsymbol{F}_{0}\right\|_{\boldsymbol{L}^{2}\left(B_{a}\right)}=O\left(a^{11 / 2}\right)
$$

Moreover, the kernels $\boldsymbol{H}_{m}(\boldsymbol{x}, \boldsymbol{\xi})$ being smooth functions for $(\boldsymbol{x}, \boldsymbol{\xi}) \in B_{a} \times B_{a}$, the corresponding integrals in $(7.3 \mathrm{a}, \mathrm{b})$, interpreted as evaluations of integral operators for $\boldsymbol{L}^{2}\left(B_{a}\right)$ densities $\boldsymbol{F}_{m}$, are estimated as

$$
\begin{gathered}
\left\|\left\langle\boldsymbol{F}_{m}, \boldsymbol{H}_{m}(\boldsymbol{x}, \cdot)\right\rangle_{B_{a}}^{\Delta \mathcal{C}}\right\|_{\boldsymbol{L}^{2}\left(B_{a}\right)} \leq C_{m}\left\|\boldsymbol{F}_{m}\right\|_{\boldsymbol{L}^{2}\left(B_{a}\right)}\left\|\boldsymbol{H}_{m}\right\|_{\boldsymbol{L}^{2}\left(B_{a} \times B_{a}\right)} \\
\left\|\left\langle\boldsymbol{F}_{m}, \boldsymbol{\nabla}_{1} \boldsymbol{H}_{m}(\boldsymbol{x}, \cdot)\right\rangle_{B_{a}}^{\Delta \mathcal{C}}\right\|_{\boldsymbol{L}^{2}\left(B_{a}\right)} \leq C_{m}\left\|\boldsymbol{F}_{m}\right\|_{\boldsymbol{L}^{2}\left(B_{a}\right)}\left\|\boldsymbol{\nabla}_{1} \boldsymbol{H}_{m}\right\|_{\boldsymbol{L}^{2}\left(B_{a} \times B_{a}\right)}
\end{gathered}
$$

for some constant $C_{m}>0$, while the kernels themselves verify

$$
\begin{aligned}
\left\|\boldsymbol{H}_{1,2}\right\|_{\boldsymbol{L}^{2}\left(B_{a} \times B_{a}\right)} & =O\left(a^{3}\right), & \left\|\boldsymbol{H}_{3}\right\|_{\boldsymbol{L}^{2}\left(B_{a} \times B_{a}\right)} & =O\left(a^{5}\right),
\end{aligned}
$$

the estimates for $\boldsymbol{H}_{3}, \boldsymbol{H}_{4}$ and $\boldsymbol{\nabla}_{1} \boldsymbol{H}_{3}$ stemming from their satisfying $\left|\boldsymbol{H}_{3}(\boldsymbol{x}, \boldsymbol{\xi})\right| \leq C a^{2},\left|\boldsymbol{H}_{4}(\boldsymbol{x}, \boldsymbol{\xi})\right| \leq C a$ and $\left|\boldsymbol{\nabla}_{1} \boldsymbol{H}_{3}(\boldsymbol{x}, \boldsymbol{\xi})\right| \leq C a$, respectively, for $(\boldsymbol{x}, \boldsymbol{\xi}) \in B_{a} \times B_{a}$ as remainders of Taylor expansions of the $C^{\infty}\left(\bar{B}_{a} \times \bar{B}_{a}\right)$ function $\boldsymbol{G}_{\mathrm{c}}$.

Using all the previous estimates in $(7.3 \mathrm{a}, \mathrm{b})$, we finally obtain

$$
\left\|\gamma_{a}\right\|_{H^{1}\left(B_{a}\right)} \leq C a^{11 / 2}
$$

for some constant $C>0$, which completes the proof of the lemma.

\section{Appendix A. Proof of Lemma 2.3}

Using $(2.20), \boldsymbol{v}_{\mathcal{B}}:=\boldsymbol{u}_{\mathcal{B}}-\boldsymbol{u}$ satisfies $\operatorname{div}\left(\mathcal{C}_{\mathcal{B}}: \varepsilon\left[\boldsymbol{v}_{\mathcal{B}}\right]\right)+\operatorname{div}\left(\Delta \mathcal{C}_{\mathcal{B}}: \varepsilon[\boldsymbol{u}]\right)=\mathbf{0}$. Taking the dot product of this equality with $\boldsymbol{v}_{\mathcal{B}}^{\prime}:=\boldsymbol{u}_{\mathcal{B}}^{\prime}-\boldsymbol{u}^{\prime}$, integrating over $\mathbb{R}^{3}$ (which is permitted since $\boldsymbol{v}_{\mathcal{B}}, \boldsymbol{v}_{\mathcal{B}}^{\prime}$ decay as $O\left(|\boldsymbol{\xi}|^{-2}\right.$ ) for $|\boldsymbol{\xi}| \rightarrow \infty$ while $\Delta \mathcal{C}_{\mathcal{B}}$ has compact support) and using the first Green identity yields

$$
\left\langle\boldsymbol{v}_{\mathcal{B}}^{\prime}, \boldsymbol{v}_{\mathcal{B}}\right\rangle_{\mathbb{R}^{3}}^{\mathcal{C}_{\mathcal{B}}}+\left\langle\boldsymbol{v}_{\mathcal{B}}^{\prime}, \boldsymbol{u}\right\rangle_{\mathcal{B}}^{\Delta \mathcal{C}}=\left\langle\boldsymbol{v}_{\mathcal{B}}^{\prime}, \boldsymbol{v}_{\mathcal{B}}\right\rangle_{\mathbb{R}^{3}}^{\mathcal{C}_{\mathcal{B}}}+\left\langle\boldsymbol{u}_{\mathcal{B}}^{\prime}, \boldsymbol{u}\right\rangle_{\mathcal{B}}^{\Delta \mathcal{C}}-\left\langle\boldsymbol{u}^{\prime}, \boldsymbol{u}\right\rangle_{\mathcal{B}}^{\Delta \mathcal{C}}=0
$$

The above identity with the roles of $\left(\boldsymbol{u}, \boldsymbol{u}_{\mathcal{B}}, \boldsymbol{v}_{\mathcal{B}}\right)$ and $\left(\boldsymbol{u}^{\prime}, \boldsymbol{u}_{\mathcal{B}}^{\prime}, \boldsymbol{v}_{\mathcal{B}}^{\prime}\right)$ reversed also holds by the same arguments. The reciprocity identity then follows from subtracting both identities together with the symmetry of $\langle\cdot, \cdot\rangle_{\mathbb{R}^{3}}^{\mathcal{C}_{\mathcal{B}}}$ and $\langle\cdot, \cdot\rangle_{\mathbb{R}^{3}}^{\Delta \mathcal{C}}$.

\section{Appendix B. EShelby SOlutions For ELLIPSOIDAl INHOMOGENEITIES}

Let $\mathcal{B}$ be an ellipsoid. Then, explicit derivations $[8,17,18,31]$ show that if $\boldsymbol{\sigma}^{\star}$ is polynomial in $\boldsymbol{x}$ with degree $p$, the restriction in $\mathcal{B}$ of the resulting displacement field $\mathcal{M}\left[\boldsymbol{\sigma}^{\star}\right](\boldsymbol{x})$ is polynomial with degree $p+1$. This in turn allows to show that FSTPs for ellipsoidal inhomogeneities and polynomial background displacements have solutions whose restriction to $\mathcal{B}$ is polynomial $[17,31]$, as recalled in Section 2.5.2. 
In particular, for densities $\boldsymbol{\sigma}^{\star}$ of the form $\boldsymbol{\sigma}^{\star}(\boldsymbol{\xi})=\mathcal{C}:\left(\boldsymbol{B}_{1}+\boldsymbol{B}_{2} \boldsymbol{\xi}\right)$, where $\boldsymbol{B}_{1}$ and $\boldsymbol{B}_{2}$ are constant second-order and third-order tensors, respectively, we have

$$
\varepsilon\left[\mathcal{M}\left[\sigma^{\star}\right]\right](\boldsymbol{x})=-\mathcal{S}^{1}(\mathcal{B}, \mathcal{C}): \boldsymbol{B}_{1}-\left(\mathcal{S}^{2}(\mathcal{B}, \mathcal{C}) \cdot \boldsymbol{B}_{2}\right) \cdot \boldsymbol{x}
$$

in terms of the (known, constant) Eshelby tensors $\mathcal{S}^{1}, \mathcal{S}^{2}[31]$ (see formulas (2.26a) when $\mathcal{B}$ is a ball).

Now, consider the FSTP in the form (2.21a) for a background displacement $\boldsymbol{u}(\boldsymbol{x})=\boldsymbol{E}_{1} \cdot \boldsymbol{x}+\frac{1}{2} \boldsymbol{E}_{2}:(\boldsymbol{x} \otimes \boldsymbol{x})$, whose strain is thus given by $\varepsilon[\boldsymbol{u}](\boldsymbol{x})=\boldsymbol{E}_{1}^{\mathrm{s}}+\boldsymbol{E}_{2}^{\mathrm{S}} \cdot \boldsymbol{x}$ (the superscript "s" again indicating symmetrization w.r.t. the first two indices). Identity (B.1) suggests to seek $\boldsymbol{u}_{\mathcal{B}}-\boldsymbol{u}$ in polynomial form, assuming the existence of tensors $\boldsymbol{B}_{1}^{\star}$ and $\boldsymbol{B}_{2}^{\star}$ such that $\boldsymbol{u}_{\mathcal{B}}-\boldsymbol{u}=\mathcal{M}\left[\boldsymbol{\sigma}^{\star}\right]$ with $\boldsymbol{\sigma}^{\star}(\boldsymbol{\xi})=\mathcal{C}:\left(\boldsymbol{B}_{1}^{\star}+\boldsymbol{B}_{2}^{\star} \cdot \boldsymbol{\xi}\right)$; this approach, known as the equivalent inclusion method $[17,26,31]$, means that the displacement perturbation induced by an ellipsoidal inhomogeneity is equated to the displacement field created in a homogeneous body by the eigenstrain $\boldsymbol{\varepsilon}^{\star}:=\boldsymbol{B}_{1}^{\star}+\boldsymbol{B}_{2}^{\star} \cdot \boldsymbol{\xi}$ applied in $\mathcal{B}$. Equation (2.21a) requires setting $\boldsymbol{\sigma}^{\star}(\boldsymbol{\xi})=-\Delta \mathcal{C}: \varepsilon\left[\boldsymbol{u}_{\mathcal{B}}\right](\boldsymbol{\xi})$, implying

$$
\Delta \mathcal{C}: \varepsilon\left[\boldsymbol{u}_{\mathcal{B}}\right](\boldsymbol{\xi})=-\mathcal{C}:\left(\boldsymbol{B}_{1}^{\star}+\boldsymbol{B}_{2}^{\star} \cdot \boldsymbol{\xi}\right) .
$$

Then, evaluating $\Delta \mathcal{C}: \varepsilon\left[\boldsymbol{u}_{\mathcal{B}}\right](\boldsymbol{\xi})$ using equation (B.1) yields

$$
\Delta \mathcal{C}: \varepsilon\left[\boldsymbol{u}_{\mathcal{B}}\right](\boldsymbol{\xi})=\Delta \mathcal{C}:\left[\boldsymbol{E}_{1}^{\mathrm{s}}+\boldsymbol{E}_{2}^{\mathrm{s}} \cdot \boldsymbol{\xi}+\mathcal{S}^{1}: \boldsymbol{B}_{1}^{\star}+\left(\mathcal{S}^{2} \cdot \boldsymbol{B}_{2}^{\star}\right) \cdot \boldsymbol{\xi}\right]
$$

Equating the above two expressions of $\Delta \mathcal{C}: \varepsilon\left[\boldsymbol{u}_{\mathcal{B}}\right](\boldsymbol{\xi})$ for any $\boldsymbol{\xi} \in \mathcal{B}$ yields the equations

$$
\text { (a) }\left(\mathcal{C}+\Delta \mathcal{C}: \mathcal{S}^{1}\right): \boldsymbol{B}_{1}^{\star}=-\Delta \mathcal{C}: \boldsymbol{E}_{1}^{\mathrm{s}}, \quad \text { (b) }\left(\widetilde{\mathcal{C}}+\Delta \mathcal{C}: \mathcal{S}^{2}\right) \cdot \boldsymbol{B}_{2}^{\star}=-\widetilde{\Delta \mathcal{C}} \cdot \boldsymbol{E}_{2}^{\mathrm{s}},
$$

with notations $\widetilde{\mathcal{C}}$ and $\widetilde{\Delta \mathcal{C}}$ defined as in (2.25). Solving (B.2a) for $\boldsymbol{B}_{1}^{\star}$ (with $\boldsymbol{E}_{1}$ given) and (B.2b) for $\boldsymbol{B}_{2}^{\star}$ (with $\boldsymbol{E}_{2}$ given), see Lemma B.1 below, and recalling the above ansatz for $\boldsymbol{u}_{\mathcal{B}}-\boldsymbol{u}$, one obtains formulas (2.25)

Lemma B.1. Equations (B.2a) and (B.2b) each have a unique solution such that $\boldsymbol{B}_{1}^{\star}=\boldsymbol{B}_{1}^{\star s}$ and $\boldsymbol{B}_{2}^{\star}=\boldsymbol{B}_{2}^{\star s}$.

Proof. Assume the existence of $\boldsymbol{B}_{1}^{\star}$ such that $\mathcal{C}: \boldsymbol{B}_{1}^{\star}=-\Delta \mathcal{C}: \mathcal{S}^{1}: \boldsymbol{B}_{1}^{\star}$. Defining $\boldsymbol{w}:=\mathcal{M}\left[\mathcal{C}: \boldsymbol{B}_{1}^{\star}\right]$, we have $\varepsilon[\mathcal{M}[\Delta \mathcal{C}: \varepsilon(\boldsymbol{w})]]=\varepsilon\left[\mathcal{M}\left(\Delta \mathcal{C}: \mathcal{S}^{1}: \boldsymbol{B}_{1}^{\star}\right)\right]=-\varepsilon\left[\mathcal{M}\left(\mathcal{C}: \boldsymbol{B}_{1}^{\star}\right)\right]=-\mathcal{S}^{1}: \boldsymbol{B}_{1}^{\star}$, implying

$$
\varepsilon[\boldsymbol{w}]+\varepsilon[\mathcal{M}(\Delta \mathcal{C}: \varepsilon[\boldsymbol{w}])]=\mathcal{S}^{1}: \boldsymbol{B}_{1}^{\star}-\mathcal{S}^{1}: \boldsymbol{B}_{1}^{\star}=\mathbf{0}
$$

Therefore, $\boldsymbol{w}$ solves equation (2.21a) for a strain-free background displacement $\boldsymbol{u}$, in which case $\boldsymbol{w}=\boldsymbol{u}$, i.e. $\boldsymbol{w}$ is strain-free, which requires $\boldsymbol{B}_{1}^{\star \mathrm{s}}=\mathbf{0}$ since $\mathcal{S}^{1}$ is known [26] to be invertible. A similar argument applies for equation (B.2b).

\section{REFERENCES}

[1] G. Allaire, F. de Gournay, F. Jouve and A.M. Toader, Structural optimization using topological and shape sensitivity via a level-set method. Control and Cybernetics 34 (2005)59-80.

[2] F. Alouges and M. Aussal, The sparse cardinal sine decomposition and its application for fast numerical convolution. Numer. Algorithms (2015) 1-22.

[3] H. Ammari, J. Garnier, V. Jugnon and H. Kang, Stability and resolution analysis for a topological derivative based imaging functional. SIAM J. Control Opt. 50 (2012) 48-76.

[4] H. Ammari and H. Kang, Polarization and moment tensors: with applications to inverse problems and effective medium theory. Vol. 162. Springer (2007).

[5] H. Ammari, H. Kang, G. Nakamura and K. Tanuma, Complete asymptotic expansions of solutions of the system of elastostatics in the presence of an inclusion of small diameter and detection of an inclusion. J. Elast. 67 (2002) 97-129.

[6] S. Amstutz and H. Andrä, A new algorithm for topology optimization using a level-set method. J. Comput. Phys. 216 (2006) 573-588.

[7] S. Andrieux and A.Ben Abda, The reciprocity gap: a general concept for flaws identification problems. Mech. Res. Commun. 20 (1993) 415-420. 
[8] R.J. Asaro and D.M. Barnett, The non-uniform transformation strain problem for an anisotropic ellipsoidal inclusion. J. Mech. Phys. Solids 23 (1975) 77-83.

[9] C. Bellis and M. Bonnet, A FEM-based topological sensitivity approach for fast qualitative identification of buried cavities from elastodynamic overdetermined boundary data. Int. J. Solids Struct. 47 (2010) 1221-1242.

[10] C. Bellis, M. Bonnet and F. Cakoni, Acoustic inverse scattering using topological derivative of far-field measurements-based $L^{2}$ cost functionals. Inverse Problems 29 (2013) 075012.

[11] M. Bonnet, Inverse acoustic scattering by small-obstacle expansion of a misfit function. Inverse Problems 24 (2008) 035022.

[12] M. Bonnet, Fast identification of cracks using higher-order topological sensitivity for 2-D potential problems. "Special issue on the advances in mesh reduction methods - In honor of Professor Subrata Mukherjee on the occasion of his 65th birthday". Eng. Anal. Boundary Elements 35 (2011) 223-235.

[13] M. Bonnet and G. Delgado, The topological derivative in anisotropic elasticity. Quarterly J. Mech. Appl. Math. 66 (2013) $557-586$.

[14] H. Brezis, Functional Analysis, Sobolev Spaces and Partial Differential Equations. 0172-5939. Springer Verlag New York (2011).

[15] P.G.Ciarlet, Linear and nonlinear functional analysis with applications. SIAM (2013).

[16] R. Dautray and J.L. Lions, Analyse mathematique et calcul numerique pour les sciences et les techniques. Masson (1984-85).

[17] J. Eshelby, The determination of the elastic field of an ellipsoidal inclusion, and related problems. Proc. Roy. Soc. 241 (1957) $376-396$.

[18] J. Eshelby, Elastic inclusions and inhomogeneities. Progress in solid Mechanics 2 (1961) 89-140.

[19] A.B. Freidin and V.A. Kucher, Solvability of the equivalent inclusion problem for an ellipsoidal inhomogeneity. Math. Mech. Solids 21 (2016) 255-262.

[20] Y. Fu, K.J. Klimkowski, G.J. Rodin, E. Berger, J.C. Browne, J.R. Singer, R.A. van der Greijn and K.S.Vemaganti, A fast solution method for the three-dimensional many-particle problems of linear elasticity. Int. J. Num. Meth. Eng. 42 (1998) $1215-1229$.

[21] S. Garreau, P. Guillaume and M. Masmoudi, The topological asymptotic for PDE systems: the elasticity case. SIAM J. Contr. Opt. 39 (2001) 1756-1778.

[22] D. Gintides and K. Kiriaki, Solvability of the integrodifferential equation of Eshelby's equivalent inclusion method. The Quarterly J. Mech. Appl. Math. 68 (2015) 85-96.

[23] M. Grédiac and F. Hild, Full-field measurements and identification in solid mechanics. John Wiley and Sons (2012).

[24] G.C. Hsiaoa and W.L. Wendland, Boundary integral equations. Springer (2008).

[25] R. Kohn and A. McKenney, Numerical implementation of a variational method for electrical impedance tomography. Inverse Problems 6 (1990) 389-414.

[26] W.P. Kuykendall, W.D. Cash, D.M. Barnetta and W.Cai, On the existence of Eshelby's equivalent ellipsoidal inclusion solution. Math. Mech. Solids 17 (2012) 840-847.

[27] P.A. Martin, Acoustic scattering by inhomogeneous obstacles. SIAM J. Appl. Math. 64 (2003) $297-308$.

[28] M. Masmoudi, J. Pommier and B. Samet, The topological asymptotic expansion for the Maxwell equations and some applications. Inverse Problems 21 (2005) 547-564.

[29] W. McLean, Strongly elliptic systems and boundary integral equations. Cambridge (2000).

[30] S.G. Mikhlin and S. Prössdorf, Singular integral operators. Vol. 1986. Springer Verlag Berlin (1986).

[31] T. Mura, Micromechanics of defects in solids. M. Nijhoff, The Hague and Boston and Higham, Mass (1982).

[32] A.G. Ramm, A simple proof of the Fredholm alternative and a characterization of the Fredholm operators. Amer. Math. Monthly. 108 (2001) 855-860.

[33] B. Samet, S. Amstutz and M. Masmoudi, The topological asymptotic for the Helmholtz equation. SIAM J. Contr. Opt. 42 (2004) 1523-1544.

[34] M. Silva, M. Matalon and D.A. Tortorelli, Higher order topological derivatives in elasticity. Inter. J. Solids Structures 47 (2010) 3053-3066.

[35] J. Sokolowski and A. Zochowski, Topological derivatives of shape functionals for elasticity systems. Mech. Based Design Struct. Machines 21 (2001) 331-349.

[36] T. Touhei, A fast volume integral equation method for elastic wave propagation in a half space. Int. J. Solids Struct. 48 (2011) 3194-3208.

[37] S. Xinchun and Lakes R.S. Stability of elastic material with negative stiffness and negative Poisson's ratio. Phys. Stat. Sol. 244 (2007) 1008-1026. 